\title{
ASYMPTOTIC PROPERTIES OF ENTROPY SOLUTIONS TO FRACTAL BURGERS EQUATION
}

\author{
NATHAEL ALIBAUD, CYRIL IMBERT, AND GRZEGORZ KARCH
}

\begin{abstract}
We study properties of solutions of the initial value problem for the nonlinear and nonlocal equation $u_{t}+\left(-\partial_{x}^{2}\right)^{\alpha / 2} u+u u_{x}=0$ with $\alpha \in(0,1]$, supplemented with an initial datum approaching the constant states $u_{ \pm}\left(u_{-}<\right.$ $\left.u_{+}\right)$as $x \rightarrow \pm \infty$, respectively. It was shown by Karch, Miao \& Xu (SIAM J. Math. Anal. 39 (2008), 1536-1549) that, for $\alpha \in(1,2)$, the large time asymptotics of solutions is described by rarefaction waves. The goal of this paper is to show that the asymptotic profile of solutions changes for $\alpha \leq 1$. If $\alpha=1$, there exists a self-similar solution to the equation which describes the large time asymptotics of other solutions. In the case $\alpha \in(0,1)$, we show that the nonlinearity of the equation is negligible in the large time asymptotic expansion of solutions.
\end{abstract}

\section{INTRODUCTION}

In this work, we continue the study of asymptotic properties of solutions of the Cauchy problem for the following nonlocal conservation law

$$
\begin{aligned}
& u_{t}+\Lambda^{\alpha} u+u u_{x}=0, \quad x \in \mathbb{R}, t>0, \\
& u(0, x)=u_{0}(x),
\end{aligned}
$$

where $\Lambda^{\alpha}=\left(-\partial^{2} / \partial x^{2}\right)^{\alpha / 2}$ is the pseudodifferential operator defined via the Fourier transform $\widehat{\left(\Lambda^{\alpha} v\right)}(\xi)=|\xi|^{\alpha} \widehat{v}(\xi)$. This equation is referred to as the fractal Burgers equation.

The initial datum $u_{0} \in L^{\infty}(\mathbb{R})$ is assumed to satisfy:

$$
\exists u_{-}<u_{+} \text {with } u_{0}-u_{-} \in L^{1}(-\infty, 0) \text { and } u_{0}-u_{+} \in L^{1}(0,+\infty)
$$

(where $u_{ \pm}$are real numbers). An interesting situation is where $u_{0} \in B V(\mathbb{R})$, that is to say

$$
u_{0}(x)=c+\int_{-\infty}^{x} m(d y)
$$

Date: October 24, 2018

2000 Mathematics Subject Classification. 35K05, 35K15.

Key words and phrases. fractal Burgers equation, asymptotic behavior of solutions, self-similar solutions, entropy solutions.

The authors would like to thank the referee for suggestions that improved significantly the presentation of the results. The first author would like to thank the Department of Mathematics Prince of Songkla University (Hat Yai campus, Thailand) for having ensured a large part of his working facilities. The second author was partially supported by the ANR project "EVOL". The work of the third author was partially supported by the European Commission Marie Curie Host Fellowship for the Transfer of Knowledge "Harmonic Analysis, Nonlinear Analysis and Probability" MTKD-CT-2004-013389, and by the Polish Ministry of Science grant N201 022 32/0902. The authors were also partially supported by PHC-Polonium project no 20078TL "Nonlinear evolutions equations with anomalous diffusions". 
with $c \in \mathbb{R}$ and a finite signed measure $m$ on $\mathbb{R}$. In that case Jourdain, Méléard, and Woyczyński [11, 12] have recently given a probabilistic interpretation to problem (1.1)-(1.2). Assumption (1.3) holds true when

$$
u_{-}=c \quad \text { and } \quad u_{+}-u_{-}=\int_{\mathbb{R}} m(d x)>0 .
$$

If $c=0$ and if $m$ is a probability measure, the function $u_{0}$ defined in (1.4) is the cumulative distribution function and this property is shared by the solution $u(t) \equiv u(\cdot, t)$ for every $t>0$ (see [11, 12]). As a consequence of our results, we describe the asymptotic behavior of the family $\{u(t)\}_{t \geq 0}$ of probability distribution functions as $t \rightarrow+\infty$ (see the summary at the end of this section).

It was shown in 14 that, under assumptions (1.3)-1.5 and for $1<\alpha \leq 2$, the large time asymptotics of solution to (1.1)-(1.2) is described by the so-called rarefaction waves. The goal of this paper is to complete these results and to obtain universal asymptotic profiles of solutions for $0<\alpha \leq 1$.

1.1. Known results. Let us first recall the results obtained in [14. For $\alpha \in$ $(1,2]$, the initial value problem for the fractal Burgers equation (1.1)-(1.2) with $u_{0} \in L^{\infty}(\mathbb{R})$ has a unique, smooth, global-in-time solution (cf. [8, Thm. 1.1], [9, Thm. 7]). If, moreover, the initial datum is of the form (1.4) and satisfies (1.3)(1.5), the corresponding solution $u$ behaves asymptotically when $t \rightarrow+\infty$ as the rarefaction wave $\left(c f . \quad\left[14\right.\right.$, Thm. 1.1]). More precisely, for every $p \in\left(\frac{3-\alpha}{\alpha-1},+\infty\right]$ there exists a constant $C>0$ such that for all $t>0$,

$$
\left\|u(t)-w^{R}(t)\right\|_{p} \leq C t^{-\frac{1}{2}\left[\alpha-1-\frac{3-\alpha}{p}\right]} \log (2+t)
$$

$\left(\|\cdot\|_{p}\right.$ is the standard norm in $\left.L^{p}(\mathbb{R})\right)$. Here, the rarefaction wave is the explicit (self-similar) function

$$
w^{R}(x, t)=w^{R}\left(\frac{x}{t}, 1\right) \equiv \begin{cases}u_{-}, & \frac{x}{t} \leq u_{-}, \\ \frac{x}{t}, & u_{-} \leq \frac{x}{t} \leq u_{+} \\ u_{+}, & \frac{x}{t} \geq u_{+}\end{cases}
$$

It is well-known that $w^{R}$ is the unique entropy solution of the Riemann problem for the nonviscous Burgers equation $w_{t}^{R}+w^{R} w_{x}^{R}=0$.

The goal of the work is to show that, for $\alpha \in(0,1]$, one should expect completely different asymptotic profiles of solutions. Let us notice that the initial value problem (1.1) - (1.2) has a unique global-in-time entropy solution for every $u_{0} \in L^{\infty}(\mathbb{R})$ and $\alpha \in(0,1]$ due to the recent work by the first author [1]. We recall this result in Section 2,

1.2. Main results. Our two main results are Theorems 1.3 and 1.6 stated below. Both of them are a consequence of the following $L^{p}$-estimate of the difference of two entropy solutions.

Theorem 1.1. Let $0<\alpha \leq 1$. Assume that $u$ and $\widetilde{u}$ are two entropy solutions of (1.1) - 1.2) with initial conditions $u_{0}$ and $\widetilde{u}_{0}$ in $L^{\infty}(\mathbb{R})$. Suppose, moreover, that $\widetilde{u}_{0}$ is non-decreasing and $u_{0}-\widetilde{u}_{0} \in L^{1}(\mathbb{R})$. Then there exists a constant $C=C(\alpha)>0$ such that for all $p \in[1,+\infty]$ and all $t>0$

$$
\|u(t)-\widetilde{u}(t)\|_{p} \leq C t^{-\frac{1}{\alpha}\left(1-\frac{1}{p}\right)}\left\|u_{0}-\widetilde{u}_{0}\right\|_{1} .
$$

Remark 1.2. (1) It is worth mentioning that this estimate is sharper than the one obtained by interpolating the $L^{1}$-contraction principle and $L^{\infty}$-bounds on the solutions. 
(2) Mention also that this result holds true for $\alpha \in(1,2]$ without additional $B V$ assumption on $u_{0}$. Consequently, as an immediate corollary of (1.6) and (1.8), one can slightly complete the results from [14. More precisely, let $\alpha \in$ $(1,2], u_{0} \in L^{\infty}(\mathbb{R})$ satisfying (1.3) and $u$ be the solution to (1.1)-(1.2). Then for every $p \in\left(\frac{3-\alpha}{\alpha-1},+\infty\right]$ there exists a constant $C>0$ such that for all $t>0$

$$
\left\|u(t)-w^{R}(t)\right\|_{p} \leq C t^{-\frac{1}{2}\left[\alpha-1-\frac{3-\alpha}{p}\right]} \log (2+t)+C t^{-\frac{1}{\alpha}\left(1-\frac{1}{p}\right)},
$$

even if $u_{0} \notin B V(\mathbb{R})$.

In the case $\alpha<1$, the linear part of the fractal Burgers equation dominates the nonlinear one for large times. In the case $\alpha=1$, both parts are balanced; indeed, self-similar solutions exist. Let us be more precise now.

For $\alpha<1$, the Duhamel principle (see equation (3.3) below) shows that the nonlinearity in equation (1.1) is negligible in the asymptotic expansion of solutions.

Theorem 1.3. (Asymptotic behavior as the linear part)

Let $0<\alpha<1$ and $u_{0} \in L^{\infty}(\mathbb{R})$ satisfying (1.3). Let $u$ be the entropy solution to (1.1)-1.2). Denote by $\left\{S_{\alpha}(t)\right\}_{t>0}$ the semi-group of linear operators whose infinitesimal generator is $-\Lambda^{\alpha}$. Consider the initial condition

$$
U_{0}(x) \equiv \begin{cases}u_{-}, & x<0, \\ u_{+}, & x>0 .\end{cases}
$$

Then, there exists a constant $C=C(\alpha)>0$ such that for all $p \in\left(\frac{1}{1-\alpha},+\infty\right]$ and all $t>0$,

$$
\begin{aligned}
\left\|u(t)-S_{\alpha}(t) U_{0}\right\|_{p} \leq & C t^{-\frac{1}{\alpha}\left(1-\frac{1}{p}\right)}\left\|u_{0}-U_{0}\right\|_{1} \\
& +C\left(u_{+}-u_{-}\right) \max \left\{\left|u_{+}\right|,\left|u_{-}\right|\right\} t^{1-\frac{1}{\alpha}\left(1-\frac{1}{p}\right)} .
\end{aligned}
$$

Remark 1.4. (1) It follows from the proof of Theorem 1.3 that inequality (1.10) is valid for every $p \in[1,+\infty]$. However, its right-hand-side decays only for $p \in\left(\frac{1}{1-\alpha},+\infty\right]$.

(2) Let us recall here the formula $S_{\alpha}(t) U_{0}=p_{\alpha}(t) * U_{0}$ where $p_{\alpha}=p_{\alpha}(x, t)$ denotes the fundamental solution of the equation $u_{t}+\Lambda^{\alpha} u=0$ (cf. the beginning of Section 3 for its properties). Hence, changing variables in the convolution $p_{\alpha}(t) * U_{0}$, one can write the asymptotic term in (1.10) in the self-similar form $\left(S_{\alpha}(t) U_{0}\right)(x)=H_{\alpha}\left(x t^{-1 / \alpha}\right)$ where $H_{\alpha}(x)=\left(p_{\alpha}(1) * U_{0}\right)(x)$ is a smooth and non-decreasing function satisfying $\lim _{x \rightarrow \pm \infty} H_{\alpha}(x)=u_{ \pm}$ and $\partial_{x} H_{\alpha}(x)=\left(u_{+}-u_{-}\right) p_{\alpha}(x, 1)$.

In the case $\alpha=1$, we use the uniqueness result from [1] combined with a standard scaling technique to show that equation (1.1) has self-similar solutions. In Section 4. we recall this well-known reasoning which leads to the proof of the following theorem.

\section{Theorem 1.5. (Existence of self-similar solutions)}

Assume $\alpha=1$. The unique entropy solution $U$ of the initial value problem (1.1)(1.2) with the initial condition (1.9) is self-similar, i.e. it has the form $U(x, t)=$ $U\left(\frac{x}{t}, 1\right)$ for all $x \in \mathbb{R}$ and all $t>0$.

Our second main convergence result states that the self-similar solution describes the large time asymptotics of other solutions to (1.1)-(1.2).

Theorem 1.6. (Asymptotic behavior as the self-similar solution) Let $\alpha=1$ and $u_{0} \in L^{\infty}(\mathbb{R})$ satisfying (1.3). Let $u$ be the entropy solution to problem 
(1.1) - (1.2). Denote by $U$ the self-similar solution from Theorem 1.5. Then there exists a constant $C=C(\alpha)>0$ such that for all $p \in[1,+\infty]$ and all $t>0$,

$$
\|u(t)-U(t)\|_{p} \leq C t^{-\left(1-\frac{1}{p}\right)}\left\|u_{0}-U_{0}\right\|_{1} .
$$

1.3. Properties of self-similar solutions. Let us complete the result stated in Theorem 1.6 by listing main qualitative properties of the profile $U(1)$.

Theorem 1.7. (Qualitative properties of the self-similar profile)

The self-similar solution from Theorem 1.5 enjoys the following properties:

p1. (Regularity) The function $U(1)=U(\cdot, 1)$ is Lipschitz-continuous.

p2. (Monotonicity and limits) $U(1)$ is increasing and satisfies

$$
\lim _{x \rightarrow \pm \infty} U(x, 1)=u_{ \pm}
$$

p3. (Symmetry) For all $y \in \mathbb{R}$, we have

$$
U(\bar{c}+y, 1)=2 \bar{c}-U(\bar{c}-y, 1) \quad \text { where } \quad \bar{c} \equiv \frac{u_{-}+u_{+}}{2} .
$$

p4. (Convex/concave) $U(1)$ is convex (resp. concave) on $(-\infty, \bar{c}]$ (resp. on $[\bar{c},+\infty))$.

p5. (Decay at infinity) We have

$$
U_{x}(x, 1) \sim \frac{u_{+}-u_{-}}{2 \pi^{2}}|x|^{-2} \quad \text { as } \quad|x| \rightarrow+\infty .
$$

Actually, the profile $U(1)$ is expected to be $C_{b}^{\infty}$ or analytic, due to recent regularity results [16, 7, 18, for the critical fractal Burgers equation with $\alpha=1$. It was shown that the solution is smooth whenever $u_{0}$ is either periodic or from $L^{2}(\mathbb{R})$ or from a critical Besov space. Unfortunately, we do not know if those results can be adapted to any initial condition from $L^{\infty}(\mathbb{R})$.

Property p3 implies that $U(x(t), t)$ is a constant equal to $\bar{c}$ along the characteristic $x(t)=\bar{c} t$, with the symmetry

$$
U(\bar{c} t+y, t)=2 \bar{c}-U(\bar{c} t-y, t)
$$

for all $t>0$ and $y \in \mathbb{R}$. Thus, the real number $\bar{c}$ can be interpreted as a mean celerity of the profile $U(t)$, which is the same mean celerity as for the rarefaction wave in (1.7).

In property p5, we obtain the decay at infinity which is the same as for the fundamental solutions $p_{1}(x, t)=t^{-1} p_{1}\left(x t^{-1}, 1\right)$ of the linear equation $u_{t}+\Lambda^{1} u=0$, given by the explicit formula

$$
p_{1}(x, 1)=\frac{2}{1+4 \pi^{2} x^{2}}
$$

Following the terminology introduced in [6], one may say that property p5 expresses a far field asymptotics and is somewhere in relation with the results in [6] for fractal conservation laws with $\alpha \in(1,2)$, where the Duhamel principle plays a crucial role. This principle is less convenient in the critical case $\alpha=1$, and our proof of p5 does not use it.

Finally, if $u_{-}=0$ and $u_{+}-u_{-}=1$, property p2 means that $U(1)$ is the cumulative distribution function of some probability law $\mathcal{L}$ with density $U_{x}(1)$. Property p3 ensures that $\mathcal{L}$ is symmetrically distributed around its median $\bar{c}$; notice that any random variable with law $\mathcal{L}$ has no expectation, because of property p5. Properties p4-p5 make precise that the density of $\mathcal{L}$ decays around $\bar{c}$ with the same rate at infinity as for the Cauchy law with density $p_{1}(x, 1)$.

The probability distributions of both laws around their respective medians can be compared as follows. 
Theorem 1.8. (Comparison with the Cauchy law)

Let $\mathcal{L}$ be the probability law with density $U_{x}(1)$, where $U$ is the self-similar solution defined in Theorem 1.5, with $u_{-}=0$ and $u_{+}=1$. Let $X$ (resp. Y) be a real random variable on some probability space $(\Omega, \mathcal{A}, \mathbb{P})$ with law $\mathcal{L}$ (resp. the Cauchy law (1.12) (with zero median)). Then, we have for all $r>0$

$$
\mathbb{P}(|X-\bar{c}|<r)<\mathbb{P}(|Y-0|<r)
$$

where $\bar{c}$ denotes the median of $X$.

Remark 1.9. More can be said in order to compare random variables $X-\bar{c}$ and $Y$. Indeed, their cumulative distribution functions satisfy $F_{X-\bar{c}}(x)=F_{Y}(x)-g(x)$ where $g$ is an explicit positive function (on the positive axis) depending the selfsimilar solution of (1.1) (see equation (6.26)).

1.4. Probabilistic interpretation of results for $\alpha \in(0,2]$. To summarize, let us emphasize the probabilistic meaning of the complete asymptotic study of the fractal Burgers equation we have now in hands. We have already mentioned that the solution $u$ of (1.1)-(1.2) supplemented with the initial datum of the form (1.4) with $c=0$ and with a probability measure $m$ on $\mathbb{R}$ is the cumulative distribution function for every $t \geq 0$. This family of probabilities defined by problem (1.1)-(1.2) behaves asymptotically when $t \rightarrow+\infty$ as

- the uniform distribution on the interval $[0, t]$ if $1<\alpha \leq 2$ (see the result from [14 recalled in inequality (1.6) above);

- the family of laws $\left\{\mathcal{L}_{t}\right\}_{t \geq 0}$ constructed in Theorem 1.5) if $\alpha=1$ (see Theorem 1.6);

- the symmetric $\alpha$-stable laws $p_{\alpha}(t)$ if $0<\alpha<1$ (cf. Theorem 1.3 and Remark [1.4).

1.5. Organization of the article. The remainder of this paper is organized as follows. In the next section, we recall the notion of entropy solutions to (1.1)(1.2) with $\alpha \in(0,1]$. Results on the regularized equation (i.e. equation (1.1) with an additional term $-\varepsilon u_{x x}$ on the left-hand-side) are gathered in Section 3 . The convergence of solutions as $\varepsilon \rightarrow 0$ to the regularized problem is discussed in Section 4 The main asymptotic results for (1.1)-(1.2) are proved in Section 5 by passage to the limit as $\varepsilon$ goes to zero. Section 6 is devoted to the qualitative study of the self-similar profile for $\alpha=1$. For the reader's convenience, sketches of proofs of a key estimate from [14 and Theorem 4.1 are given in appendices; the technical lemmata are also gathered in appendices.

\section{ENTROPY SOLUTIONS FOR $0<\alpha \leq 1$}

2.1. Lévy-Khintchine's representation of $\Lambda^{\alpha}$. It is well-known that the operator $\Lambda^{\alpha}=\left(-\partial^{2} / \partial x^{2}\right)^{\alpha / 2}$ for $\alpha \in(0,2)$ has an integral representation: for every Schwartz function $\varphi \in \mathcal{S}(\mathbb{R})$ and each $r>0$, we have

$$
\Lambda^{\alpha} \varphi=\Lambda_{r}^{(\alpha)} \varphi+\Lambda_{r}^{(0)} \varphi
$$

where the integro-differential operators $\Lambda_{r}^{(\alpha)}$ and $\Lambda_{r}^{(0)}$ are defined by

$$
\begin{aligned}
& \Lambda_{r}^{(\alpha)} \varphi(x) \equiv-G_{\alpha} \int_{|z| \leq r} \frac{\varphi(x+z)-\varphi(x)-\varphi_{x}(x) z}{|z|^{1+\alpha}} d z, \\
& \Lambda_{r}^{(0)} \varphi(x) \equiv-G_{\alpha} \int_{|z|>r} \frac{\varphi(x+z)-\varphi(x)}{|z|^{1+\alpha}} d z,
\end{aligned}
$$

where $G_{\alpha} \equiv \frac{\alpha \Gamma\left(\frac{1+\alpha}{2}\right)}{2 \pi^{\frac{1}{2}+\alpha} \Gamma\left(1-\frac{\alpha}{2}\right)}>0$ and $\Gamma$ is Euler's function. On the basis of this formula, we can extend the domain of definition of $\Lambda^{\alpha}$ and consider $\Lambda_{r}^{(0)}$ and $\Lambda_{r}^{(\alpha)}$ 
as the operators

$$
\Lambda_{r}^{(0)}: C_{b}(\mathbb{R}) \rightarrow C_{b}(\mathbb{R}) \text { and } \Lambda_{r}^{(\alpha)}: C_{b}^{2}(\mathbb{R}) \rightarrow C_{b}(\mathbb{R}) ;
$$

hence, $\Lambda^{\alpha}: C_{b}^{2}(\mathbb{R}) \rightarrow C_{b}(\mathbb{R})$.

Let us recall some properties on these operators. First, the so-called Kato inequality can be generalized to $\Lambda^{\alpha}$ for each $\alpha \in(0,2]$ : let $\eta \in C^{2}(\mathbb{R})$ be convex and $\varphi \in C_{b}^{2}(\mathbb{R})$, then

$$
\Lambda^{\alpha} \eta(u) \leq \eta^{\prime}(u) \Lambda^{\alpha} u
$$

Note that for $\alpha=2$ we have

$$
-(\eta(u))_{x x}=-\eta^{\prime \prime}(u) u_{x}^{2}-\eta^{\prime}(u) u_{x x} \leq-\eta^{\prime}(u) u_{x x} \text { since } \eta^{\prime \prime} \geq 0 .
$$

If $\alpha \in(0,2)$, inequality (2.4) is the direct consequence of the integral representation (2.1) - (2.3) and of the following inequalities

$$
\Lambda_{r}^{(0)} \eta(u) \leq \eta^{\prime}(u) \Lambda_{r}^{(0)} u \quad \text { and } \quad \Lambda_{r}^{(\alpha)} \eta(u) \leq \eta^{\prime}(u) \Lambda_{r}^{(\alpha)} u,
$$

resulting from the convexity of the function $\eta$.

Finally, these operators satisfy the integration by parts formula: for all $u \in$ $C_{b}^{2}(\mathbb{R})$ and $\varphi \in \mathcal{D}(\mathbb{R})$, we have

$$
\int_{\mathbb{R}} \varphi \Lambda u d x=\int_{\mathbb{R}} u \Lambda \varphi d x
$$

where $\Lambda \in\left\{\Lambda_{r}^{(0)}, \Lambda_{r}^{(\alpha)}, \Lambda^{\alpha}\right\}$ for every $\alpha \in(0,2]$ and all $r>0$. Notice that $\Lambda \varphi \in$ $L^{1}(\mathbb{R})$, since it is obvious from (2.2)-(2.3) that $\Lambda_{r}^{(\alpha)}: W^{2,1}(\mathbb{R}) \rightarrow L^{1}(\mathbb{R})$ and $\Lambda_{r}^{(0)}$ : $L^{1}(\mathbb{R}) \rightarrow L^{1}(\mathbb{R})$.

Detailed proofs of all these properties are based on the representation (2.1)-(2.3) and are written e.g. in [1].

2.2. Existence and uniqueness of entropy solutions. It was shown in [2] (see also [16]) that solutions of the initial value problem for the fractal conservation law

$$
\begin{aligned}
& u_{t}+\Lambda^{\alpha} u+(f(u))_{x}=0, \quad x \in \mathbb{R}, t>0, \\
& u(0, x)=u_{0}(x),
\end{aligned}
$$

where $f: \mathbb{R} \rightarrow \mathbb{R}$ is locally Lipschitz-continuous, can become discontinuous in finite time if $0<\alpha<1$. Hence, in order to deal with discontinuous solutions, the notion of entropy solution in the sense of Kruzhkov was extended in [1] to fractal conservation laws (2.7)-(2.8) (see also [15] for the recent generalization to Lévy mixed hyperbolic/parabolic equations). Here, the crucial role is played by the Lévy-Khintchine's representation (2.1)-(2.3) of the operator $\Lambda^{\alpha}$.

Definition 2.1. Let $0<\alpha \leq 1$ and $u_{0} \in L^{\infty}(\mathbb{R})$. A function $u \in L^{\infty}(\mathbb{R} \times(0,+\infty))$ is an entropy solution to (2.7)-(2.8) if for all $\varphi \in \mathcal{D}(\mathbb{R} \times[0,+\infty)), \varphi \geq 0, \eta \in C^{2}(\mathbb{R})$ convex, $\phi: \mathbb{R} \rightarrow \mathbb{R}$ such that $\phi^{\prime}=\eta^{\prime} f^{\prime}$, and $r>0$, we have

$$
\begin{aligned}
\int_{\mathbb{R}} \int_{0}^{+\infty}\left(\eta(u) \varphi_{t}+\phi(u) \varphi_{x}-\eta(u) \Lambda_{r}^{(\alpha)} \varphi\right. & \left.-\varphi \eta^{\prime}(u) \Lambda_{r}^{(0)} u\right) d x d t \\
& +\int_{\mathbb{R}} \eta\left(u_{0}(x)\right) \varphi(x, 0) d x \geq 0 .
\end{aligned}
$$

Note that, due to formula (2.3), the quantity $\Lambda_{r}^{(0)} u$ in the above inequality is well-defined for any bounded function $u$.

The notion of entropy solutions allows us to solve the fractal Burgers equation for the range of exponent $\alpha \in(0,1]$. 
Theorem 2.2 ([1]). Assume that $0<\alpha \leq 1$ and $u_{0} \in L^{\infty}(\mathbb{R})$. There exists a unique entropy solution $u$ to problem (2.7)-(2.8). This solution $u$ belongs to $C\left([0,+\infty) ; L_{\text {loc }}^{1}(\mathbb{R})\right)$ and satisfies $u(0)=u_{0}$. Moreover, we have the following maximum principle: $\operatorname{ess} \inf u_{0} \leq u \leq \operatorname{ess} \sup u_{0}$.

If $\alpha \in(1,2]$, all solutions to (2.7)-(2.8) with bounded initial conditions are smooth and global-in-time (see [8, 16, 17]). On the other hand, the occurrence of discontinuities in finite time of entropy solutions to (2.7)-(2.8) with $\alpha=1$ seems to be unclear. As mentioned in the introduction, regularity results have recently been obtained [16, 17, 18, for a large class of initial conditions which, unfortunately, does not include general $L^{\infty}$-initial data. Nevertheless, Theorem 2.2 provides the existence and the uniqueness of a global-in-time entropy solution even for the critical case $\alpha=1$.

\section{Regularized Problem}

In this section, we gather properties of solutions to the Cauchy problem for the regularized fractal Burgers equation with $\varepsilon>0$

$$
\begin{aligned}
& u_{t}^{\varepsilon}+\Lambda^{\alpha} u^{\varepsilon}-\varepsilon u_{x x}^{\varepsilon}+u^{\varepsilon} u_{x}^{\varepsilon}=0, \quad x \in \mathbb{R}, t>0, \\
& u^{\varepsilon}(x, 0)=u_{0}(x) .
\end{aligned}
$$

Our purpose is to derive asymptotic stability estimates of a solution $u^{\varepsilon}=u^{\varepsilon}(x, t)$ (uniform in $\varepsilon$ ) that will be valid for (1.1) (1.2) after passing to the limit $\varepsilon \rightarrow 0$. Most of the results of this section are based on a key estimate from 14]; unfortunately, this estimate is not explicitely stated as a lemma in [14. Hence, for the sake of completeness, we have recalled this key estimate in Lemma A.1 in Appendix A as well as the main lines of its proof.

Below, we will use the following integral formulation of the initial value problem (3.1)-3.2

$$
u^{\varepsilon}(t)=S_{\alpha}^{\varepsilon}(t) u_{0}-\int_{0}^{t} S_{\alpha}^{\varepsilon}(t-\tau) u^{\varepsilon}(\tau) u_{x}^{\varepsilon}(\tau) d \tau,
$$

where $\left\{S_{\alpha}^{\varepsilon}(t)\right\}_{t>0}$ is the semi-group generated by $-\Lambda^{\alpha}+\varepsilon \partial_{x}^{2}$.

If, for each $\alpha \in(0,2]$, the function $p_{\alpha}$ denotes the fundamental solution of the linear equation $u_{t}+\Lambda^{\alpha} u=0$, then

$$
S_{\alpha}^{\varepsilon}(t) u_{0}=p_{\alpha}(t) * p_{2}(\varepsilon t) * u_{0}
$$

It is well-known that $p_{\alpha}=p_{\alpha}(x, t)$ can be represented via the Fourier transform (w.r.t. the $x$-variable) $\widehat{p}_{\alpha}(\xi, t)=e^{-t|\xi|^{\alpha}}$. In particular,

$$
p_{\alpha}(x, t)=t^{-\frac{1}{\alpha}} P_{\alpha}\left(x t^{-\frac{1}{\alpha}}\right),
$$

where $P_{\alpha}$ is the inverse Fourier transform of $e^{-|\xi|^{\alpha}}$. For every $\alpha \in(0,2]$ the function $P_{\alpha}$ is smooth, non-negative, $\int_{\mathbb{R}} P_{\alpha}(y) d y=1$, and satisfies the estimates (optimal for $\alpha \neq 2$ )

$$
0<P_{\alpha}(x) \leq C(1+|x|)^{-(\alpha+1)} \text { and }\left|\partial_{x} P_{\alpha}\right| \leq C(1+|x|)^{-(\alpha+2)}
$$

for a constant $C$ and all $x \in \mathbb{R}$.

One can see that problem (3.1) - (3.2) admits a unique global-in-time smooth solution.

Theorem $3.1([8])$. Let $\alpha \in(0,2], \varepsilon>0$ and $u_{0} \in L^{\infty}(\mathbb{R})$. There exists a unique solution $u^{\varepsilon}$ to problem (3.1) (3.2) in the following sense:

- $u^{\varepsilon} \in C_{b}(\mathbb{R} \times(0,+\infty)) \cap C_{b}^{\infty}(\mathbb{R} \times(a,+\infty))$ for all $a>0$,

- $u^{\varepsilon}$ satisfies equation (3.1) on $\mathbb{R} \times(0,+\infty)$,

- $\lim _{t \rightarrow 0} u^{\varepsilon}(t)=u_{0}$ in $L^{\infty}(\mathbb{R})$ weak-* and in $L_{l o c}^{p}(\mathbb{R})$ for all $p \in[1,+\infty)$. 
Moreover, the following maximum principle holds true:

$$
\text { ess inf } u_{0} \leq u^{\varepsilon} \leq \operatorname{ess} \sup u_{0} .
$$

Proof. Here, the results from [8] can be easily modified in order to get the existence and the regularity of solutions to (3.1)-(3.2) with $\varepsilon>0$.

Here are some elementary properties (comparison principle, $L^{1}$-contraction principle and non-increase of the $B V$-semi-norm) of fractal conservation laws that will be needed.

Proposition $3.2([8])$. Let $\varepsilon>0$ and $u^{\varepsilon}$ and $\widetilde{u^{\varepsilon}}$ be solutions to (3.1)-(3.2) with respective initial data $u_{0}$ and $\widetilde{u_{0}}$ in $L^{\infty}(\mathbb{R})$. Then:

- if $u_{0} \leq \widetilde{u_{0}}$ then $u^{\varepsilon} \leq \widetilde{u^{\varepsilon}}$,

- if $u_{0}-\widetilde{u_{0}} \in L^{1}(\mathbb{R})$ then $\left\|u^{\varepsilon}-\widetilde{u^{\varepsilon}}\right\|_{L^{\infty}\left(0,+\infty, L^{1}\right)} \leq\left\|u_{0}-\widetilde{u_{0}}\right\|_{1}$,

- if $u_{0} \in B V(\mathbb{R})$ then $\left\|u_{x}^{\varepsilon}(t)\right\|_{L^{\infty}\left(0,+\infty, L^{1}\right)} \leq\left|u_{0}\right|_{B V}$

where $\|\cdot\|_{L^{\infty}\left(0,+\infty, L^{1}\right)}$ and $|\cdot|_{B V}$ denote respectively the norm in $L^{\infty}\left(0,+\infty, L^{1}(\mathbb{R})\right)$ and the semi-norm in $B V(\mathbb{R})$.

Sketch of the proof. As explained in [8, Remarks $1.2 \& 6.2]$, these properties are immediate consequences of the splitting method developped in [8] and the facts that both the hyperbolic equation $u_{t}+u u_{x}=0$ and the fractal equation $u_{t}+\Lambda^{\alpha} u-\varepsilon u_{x x}=$ 0 satisfy these properties.

The next proposition provides an estimate on the gradient of $u^{\varepsilon}$.

Proposition 3.3. Let $0<\alpha \leq 1$ and $u_{0} \in L^{\infty}(\mathbb{R})$ be non-decreasing. For each $\varepsilon>$ 0 , denote by $u^{\varepsilon}$ the solution to (3.1) 3.2. Then:

- $u_{x}^{\varepsilon}(x, t) \geq 0$ for all $x \in \mathbb{R}$ and $t>0$,

- there exists a constant $C=C(\alpha)>0$ such that for all $\varepsilon>0, p \in[1,+\infty]$ and $t>0$,

$$
\left\|u_{x}^{\varepsilon}(t)\right\|_{p} \leq C t^{-\frac{1}{\alpha}\left(1-\frac{1}{p}\right)}\left|u_{0}\right|_{B V} .
$$

Proof. For any fixed real $h$, the function $u^{\varepsilon}(\cdot+h, \cdot)$ is the solution to (3.1)-(3.2) with the initial datum $u_{0}(\cdot+h)$. Consequently, for non-decreasing $u_{0}$ and for $h>0$, the inequality $u_{0}(\cdot+h) \geq u_{0}(\cdot)$ and the comparison principle imply $u^{\varepsilon}(\cdot+h, \cdot) \geq u^{\varepsilon}(\cdot, \cdot)$ which gives $u_{x}^{\varepsilon} \geq 0$.

To show the decay of the $L^{p}$-norm, one slightly modifies the arguments from [14, Proof of Lemma 3.1]. One shall use Lemma A.1 with $v \equiv u_{x}^{\varepsilon}$. It is clear that $v$ satisfies the required regularity: for all $a>0$

$$
v \in C_{b}^{\infty}(\mathbb{R} \times(a,+\infty)) \cap L^{\infty}\left(0,+\infty, L^{1}(\mathbb{R})\right),
$$

thanks to Proposition 3.2 ensuring that

$$
\|v\|_{L^{\infty}\left(0,+\infty, L^{1}\right)}=\left\|u_{x}^{\varepsilon}\right\|_{L^{\infty}\left(0,+\infty, L^{1}\right)} \leq\left|u_{0}\right|_{B V} .
$$

It thus rests to show that $v$ satisfies (A.1). By interpolation of the inequality above and the $L^{\infty}$-bound on $v$ from Theorem 3.1, one sees that for all $p \in[1,+\infty]$ and all $t>0$,

$$
v(t) \in L^{p}(\mathbb{R}) \quad \text { and } \quad v_{t}(t), \Lambda^{\alpha} v(t), v_{x}(t), v_{x x}(t) \in L^{\infty}(\mathbb{R}) .
$$

Hence, for $p \in[2,+\infty)$, one can multiply the equation for $v$

$$
v_{t}+\Lambda^{\alpha} v-\varepsilon v_{x x}+\left(u^{\varepsilon} u_{x}^{\varepsilon}\right)_{x}=0
$$

by $v^{p-1}$ to obtain after integration:

$$
\int_{\mathbb{R}} v_{t} v^{p-1} d x+\int_{\mathbb{R}} v^{p-1} \Lambda^{\alpha} v d x-\varepsilon \int_{\mathbb{R}} v_{x x} v^{p-1} d x+\frac{p-1}{p} \int_{\mathbb{R}} v^{p+1} d x=0 ;
$$


here one has used that $\lim _{|x| \rightarrow+\infty} v(x, t)=0$ (since $v(t) \in C_{b}^{\infty}(\mathbb{R}) \cap L^{1}(\mathbb{R})$ ) to drop the boundary terms providing from integration by parts. Integrating again by parts, one sees that

$$
-\varepsilon \int_{\mathbb{R}} v_{x x} \Phi(v) d x=\varepsilon \int_{\mathbb{R}} v_{x}^{2} \Phi^{\prime}(v) d x \geq 0
$$

for all non-decreasing function $\Phi \in C^{1}(\mathbb{R})$ with $\Phi(0)=0$; Choosing $\Phi(v)=|v|^{p-2} v$, one gets

$$
-\varepsilon \int_{\mathbb{R}} v_{x x}|v|^{p-2} v d x \geq 0
$$

We deduce from (3.9), (3.10) and the non-negativity of $v$ that

$$
\int_{\mathbb{R}} v_{t}|v|^{p-2} v d x+\int_{\mathbb{R}}|v|^{p-2} v \Lambda^{\alpha} v d x \leq 0
$$

for all $p \in[2,+\infty)$ and $t>0$. This is exactly the required differential inequation in (A.1). Lemma A.1 thus completes the proof.

We can now give asymptotic stability estimates uniform in $\varepsilon$.

Theorem 3.4. Let $\alpha \in(0,2]$. Consider two initial data $u_{0}$ and $\widetilde{u}_{0}$ in $L^{\infty}(\mathbb{R})$ such that $\widetilde{u}_{0}$ is non-decreasing and $u_{0}-\widetilde{u}_{0} \in L^{1}(\mathbb{R})$. For each $\varepsilon>0$, denote by $u^{\varepsilon}$ and $\widetilde{u^{\varepsilon}}$ the corresponding solutions to (3.1)-(3.2). Then, there exists a constant $C=C(\alpha)>0$ such for all $\varepsilon>0, p \in[1,+\infty]$ and $t>0$

$$
\left\|u^{\varepsilon}(t)-\widetilde{u^{\varepsilon}}(t)\right\|_{p} \leq C t^{-\frac{1}{\alpha}\left(1-\frac{1}{p}\right)}\left\|u_{0}-\widetilde{u}_{0}\right\|_{1} .
$$

Proof. The proof follows the arguments from [14, Proof of Lemma 3.1] by skipping the additional term providing from $-\varepsilon u_{x x}^{\varepsilon}$. That is to say, one uses again Lemma A.1 with $v=u^{\varepsilon}-\widetilde{u^{\varepsilon}}$. First, the $L^{1}$-contraction principle (see Proposition 3.2) ensures that $v$ satisfies the required regularity with

$$
\left\|u^{\varepsilon}-\widetilde{u^{\varepsilon}}\right\|_{L^{\infty}\left(0,+\infty, L^{1}\right)} \leq\left\|u_{0}-\widetilde{u_{0}}\right\|_{1} .
$$

In particular, once again the interpolation of the $L^{1}$ - and $L^{\infty}$-norms implies that $v$ is $L^{p}$ in space for all time and all $p \in[1,+\infty]$. Second, one takes $p \in[2,+\infty)$ (so that all the integrands below are integrable) and one multiplies the difference of the equations satisfied by $u^{\varepsilon}$ and $\widetilde{u^{\varepsilon}}$ by $|v|^{p-2} v$. One gets after integration:

$$
\begin{aligned}
\int_{\mathbb{R}} v_{t}|v|^{p-2} v d x & +\int_{\mathbb{R}}|v|^{p-2} v \Lambda^{\alpha} v d x \\
& -\varepsilon \int_{\mathbb{R}} v_{x x}|v|^{p-2} v d x+\frac{1}{2} \int_{\mathbb{R}}\left(v^{2}+2 v \widetilde{u^{\varepsilon}}\right)_{x}|v|^{p-2} v d x=0 .
\end{aligned}
$$

The last term of the left-hand side of this equality is non-negative, since integrations by parts give

$$
\begin{aligned}
& \int_{\mathbb{R}}\left(v^{2}+2 v \widetilde{u^{\varepsilon}}\right)_{x}|v|^{p-2} v d x \\
& =\int_{\mathbb{R}} 2 v_{x}|v|^{p} d x+\int_{\mathbb{R}} 2 \widetilde{u^{\varepsilon}} v_{x}|v|^{p-2} v d x+\int_{\mathbb{R}} 2 \widetilde{u_{x}^{\varepsilon}}|v|^{p} d x, \\
& =2\left(1-\frac{1}{p}\right) \int_{\mathbb{R}} 2 \widetilde{u_{x}^{\varepsilon}}|v|^{p} d x \geq 0
\end{aligned}
$$

(once again the boundary terms can be skipped since $v$ vanishes for large $x$ ). Moreover the third term of (3.12) is also non-negative by (3.10). One easily deduces the desired inequality (A.1) and completes the proof by Lemma A.1. 
Theorem 3.5. Let $0<\alpha<1$ and $u_{0} \in L^{\infty}(\mathbb{R})$ be non-decreasing. For each $\varepsilon>0$, denote by $u^{\varepsilon}$ the solution to (3.1)-(3.2). Then, there exists $C=C(\alpha)>0$ such that for all $\varepsilon>0, p \in[1,+\infty]$ and $t>0$

$$
\left\|u^{\varepsilon}(t)-S_{\alpha}^{\varepsilon}(t) u_{0}\right\|_{p} \leq C\left\|u_{0}\right\|_{\infty}\left|u_{0}\right|_{B V} t^{1-\frac{1}{\alpha}\left(1-\frac{1}{p}\right)}
$$

(where $\left\{S_{\alpha}^{\varepsilon}(t)\right\}_{t>0}$ is generated by $-\Lambda^{\alpha}+\varepsilon \partial_{x}^{2}$ ).

Proof. Using the integral equation (3.3) we immediately obtain

$$
\left\|u^{\varepsilon}(t)-S_{\alpha}^{\varepsilon}(t) u_{0}\right\|_{p} \leq \int_{0}^{t}\left\|S_{\alpha}^{\varepsilon}(t-\tau) u^{\varepsilon}(\tau) u_{x}^{\varepsilon}(\tau)\right\|_{p} d \tau .
$$

Now, we estimate the integral in the right-hand side of (3.13) using the $L^{p}$-decay of the semi-group $S_{\alpha}^{\varepsilon}(t)$ as well as inequalities (3.7) and (3.8). Indeed, it follows from (3.5)- (3.6) that

$$
\left\|p_{2}(\varepsilon t)\right\|_{1}=1 \quad \text { and } \quad\left\|p_{\alpha}(t)\right\|_{r}=t^{-\frac{1}{\alpha}\left(1-\frac{1}{r}\right)}\left\|p_{\alpha}(1)\right\|_{r}
$$

for every $r \in[1,+\infty]$. Hence, by the Young inequality for the convolution and inequalities (3.7), (3.8), we obtain

$$
\begin{aligned}
\| S_{\alpha}^{\varepsilon}(t-\tau) & u^{\varepsilon}(\tau) u_{x}^{\varepsilon}(\tau) \|_{p} \\
& \leq\left\|p_{\alpha}(t-\tau) *\left(u^{\varepsilon}(\tau) u_{x}^{\varepsilon}(\tau)\right)\right\|_{p}, \\
& \leq C(t-\tau)^{-\frac{1}{\alpha}\left(\frac{1}{q}-\frac{1}{p}\right)}\left\|u^{\varepsilon}(\tau)\right\|_{\infty}\left\|u_{x}^{\varepsilon}(\tau)\right\|_{q}, \\
& \leq C(t-\tau)^{-\frac{1}{\alpha}\left(\frac{1}{q}-\frac{1}{p}\right)}\left\|u_{0}\right\|_{\infty}\left|u_{0}\right|_{B V} \tau^{-\frac{1}{\alpha}\left(1-\frac{1}{q}\right)},
\end{aligned}
$$

for all $1 \leq q \leq p \leq+\infty, t>0, \tau \in(0, t)$, where the constant $C$ only depends on $\max _{r \in[1,+\infty]}\left\|p_{\alpha}(1)\right\|_{r}$ and the constant in (3.8).

Next, we decompose the integral on the right-hand side of (3.13) as follows $\int_{0}^{t} \ldots d \tau=\int_{0}^{t / 2} \ldots d \tau+\int_{t / 2}^{t} \ldots d \tau$ and we bound both integrands by using inequality (3.14) either with $q=1$ or with $q=p$. This leads to the following inequality

$$
\begin{aligned}
& \left\|u^{\varepsilon}(t)-S_{\alpha}^{\varepsilon}(t) u_{0}\right\|_{p} \\
& \leq C\left\|u_{0}\right\|_{\infty}\left|u_{0}\right|_{B V}\left(\int_{0}^{t / 2}(t-\tau)^{-\frac{1}{\alpha}\left(1-\frac{1}{p}\right)} d \tau+\int_{t / 2}^{t} \tau^{-\frac{1}{\alpha}\left(1-\frac{1}{p}\right)} d \tau\right), \\
& \quad=C\left\|u_{0}\right\|_{\infty}\left|u_{0}\right|_{B V} \frac{2^{\beta}-1}{\beta 2^{\beta-1}} t^{\beta},
\end{aligned}
$$

where $\beta \equiv 1-\frac{1}{\alpha}\left(1-\frac{1}{p}\right)$. It is readily seen that $\beta \in \mathbb{R} \rightarrow \frac{2^{\beta}-1}{\beta 2^{\beta-1}}$ is positive and continuous and that $p \in[1,+\infty) \rightarrow 1-\frac{1}{\alpha}\left(1-\frac{1}{p}\right)$ is bounded. This completes the proof of Theorem 3.5

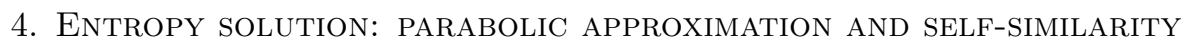

In this section, we state the result on the convergence, as $\varepsilon \rightarrow 0$, of solutions $u^{\varepsilon}$ of (3.1) - (3.2) toward the entropy solution $u$ of (1.1)-(1.2). We also prove Theorem 1.5 about self-similar entropy solutions in the case $\alpha=1$.

Together with the general fractal conservation law (2.7)-(2.8), we study the associated regularized problem

$$
\begin{aligned}
& u_{t}^{\varepsilon}+\Lambda^{\alpha} u^{\varepsilon}-\varepsilon u_{x x}^{\varepsilon}+\left(f\left(u^{\varepsilon}\right)\right)_{x}=0, \quad x \in \mathbb{R}, t>0, \\
& u^{\varepsilon}(x, 0)=u_{0}(x)
\end{aligned}
$$

where $f \in C^{\infty}(\mathbb{R})$. Hence, by results of [8] (see also Theorem 3.1), problem (4.1)(4.2) admits a unique, global-in-time, smooth solution $u^{\varepsilon}$. 
Theorem 4.1. Let $u_{0} \in L^{\infty}(\mathbb{R})$. For each $\varepsilon>0$, let $u^{\varepsilon}$ be the solution to (4.1)(4.2) and $u$ be the entropy solution to (2.7)-(2.8). Then, for every $T>0, u^{\varepsilon} \rightarrow u$ in $C\left([0, T] ; L_{l o c}^{1}(\mathbb{R})\right)$ as $\varepsilon \rightarrow 0$.

The proof of Theorem 4.1 is given in Appendix B

Remark 4.2. This result actually holds true for only locally Lipschitz-continuous fluxes $f$. More generally, multidimensional fractal conservation laws with source terms $h=h(u, x, t)$ and fluxes $f=f(u, x, t)$ (see 9, 8]) can be considered.

Proof of Theorem 1.5. The existence of the solution $U$ to equation (1.1) with $\alpha=1$ supplemented with the initial condition (1.9) is provided by Theorem 2.2. To obtain the self-similar form of $U$, we follow a standard argument based on the uniqueness result from Theorem 2.2, Observe that if $U$ is the solution to (1.1), the rescaled function $U^{\lambda}(x, t)=U(\lambda x, \lambda t)$ is the solution for every $\lambda>0$, too. Since, the initial datum (1.9) is invariant under the rescaling $U_{0}^{\lambda}(x)=U_{0}(\lambda x)$, by the uniqueness, we obtain that for all $\lambda>0, U(x, t)=U(\lambda x, \lambda t)$ for a.e. $(x, t) \in \mathbb{R} \times(0,+\infty)$.

\section{Passage to the limit $\varepsilon \rightarrow 0$ And asymptotic study}

In this section, we prove Theorems 1.1, 1.3 and 1.6.

Proof of Theorem 1.1. Denote by $u^{\varepsilon}$ and $\widetilde{u}^{\varepsilon}$ the solutions to the regularized equation (3.1) with the initial conditions $u_{0}$ and $\widetilde{u}_{0}$. By Theorem 4.1 and the maximum principle (3.7), we know that $\lim _{\varepsilon \rightarrow 0} u^{\varepsilon}(t)=u(t)$ and $\lim _{\varepsilon \rightarrow 0} \widetilde{u^{\varepsilon}}(t)=\widetilde{u}(t)$ in $L_{l o c}^{p}(\mathbb{R})$ for every $p \in[1,+\infty)$ and in $L^{\infty}(\mathbb{R})$ weak-*. Hence, for each $R>0$ and $p \in[1,+\infty]$, using Theorem 3.4 we have

$$
\begin{aligned}
\|u(t)-\widetilde{u}(t)\|_{L^{p}((-R, R))} & \leq \liminf _{\varepsilon \rightarrow 0}\left\|u^{\varepsilon}(t)-\widetilde{u}^{\varepsilon}(t)\right\|_{L^{p}((-R, R))} \\
& \leq C t^{-\frac{1}{\alpha}\left(1-\frac{1}{p}\right)}\left\|u_{0}-\widetilde{u}_{0}\right\|_{1} .
\end{aligned}
$$

Since $R>0$ is arbitrary and the right-hand side of this inequality does not depend on $R$, we complete the proof of inequality (1.8).

Proof of Theorem 1.3. In view of Theorem 1.1, it suffices to show the following inequality

$$
\left\|\widetilde{u}(t)-S_{\alpha}(t) u_{0}\right\|_{p} \leq C\left\|U_{0}\right\|_{\infty}\left|U_{0}\right|_{B V} t^{1-\frac{1}{\alpha}\left(1-\frac{1}{p}\right)},
$$

where $\widetilde{u}$ is the solution to (1.1) with $U_{0}$ as the initial condition. Notice that $\left\|U_{0}\right\|_{\infty}=u_{+}-u_{-}$and $\left|U_{0}\right|_{B V}=\max \left\{\left|u_{+}\right|,\left|u_{-}\right|\right\}$in this case.

Here, we argue exactly as in the proof of Theorem 1.1, since we can assume that $\lim _{\varepsilon \rightarrow 0} \widetilde{u}^{\varepsilon}(t)=\widetilde{u}(t)$ in $L_{l o c}^{p}(\mathbb{R})$ for every $p \in[1,+\infty)$ and in $L^{\infty}(\mathbb{R})$ weak-*. Moreover, it is well-known that for fixed $t>0$

$$
\lim _{\varepsilon \rightarrow 0} S_{\alpha}^{\varepsilon}(t) U_{0}=\lim _{\varepsilon \rightarrow 0} p_{2}(\varepsilon t) *\left(p_{\alpha}(t) * U_{0}\right)=S_{\alpha}(t) U_{0} \quad \text { in } \quad L^{p}(\mathbb{R})
$$

for all $p \in[1,+\infty]$. Hence, for every $R>0$ and $p \in[1,+\infty]$, by Theorem 3.5. we obtain

$$
\begin{aligned}
\left\|\widetilde{u}(t)-S_{\alpha}(t) U_{0}\right\|_{L^{p}((-R, R))} & \leq \liminf _{\varepsilon \rightarrow 0}\left\|\widetilde{u}^{\varepsilon}(t)-S_{\alpha}^{\varepsilon}(t) U_{0}\right\|_{L^{p}((-R, R))} \\
& \leq C\left\|U_{0}\right\|_{\infty}\left|U_{0}\right|_{B V} t^{1-\frac{1}{\alpha}\left(1-\frac{1}{p}\right)} .
\end{aligned}
$$

The proof is completed by letting $R \rightarrow+\infty$.

Proof of Theorem 1.6. Apply Theorem 1.1 with $\alpha=1$ and $\widetilde{u}_{0}=U_{0}$.

6. Qualitative Study OF THE SElF-SIMILAR PROFILE FOR $\alpha=1$

This section is devoted to the proof of Theorems 1.7 and 1.8 , 
6.1. Proof of properties p1-p4 from Theorem 1.7. The Lipschitz-continuity stated in $\mathrm{p} 1$ is an immediate consequence of Proposition 3.3 and Theorem 4.1 Indeed, $U(1)$ is the limit in $L_{\text {loc }}^{1}(\mathbb{R})$ of $u^{\varepsilon}(1)$ as $\varepsilon \rightarrow 0$, where $u^{\varepsilon}$ is solution to (3.1)(3.2) with $u_{0}=U_{0}$ defined in (1.9). Moreover, by (3.8), the family $\left\{u^{\varepsilon}(1): \varepsilon>0\right\}$ is equi-Lipschitz-continuous, which implies that the limit $U(1)$ is Lipschitz-continuous.

Before proving properties p2-p4, let us reduce the problem to a simpler one. We remark that equation (1.1) is invariant under the transformation

$$
V(x, t) \equiv U(x+\bar{c} t, t)-\bar{c} \quad \text { where } \quad \bar{c} \equiv \frac{u_{-}+u_{+}}{2} ;
$$

that is to say, if $U$ is a solution to (1.1) with $U(x, 0)=U_{0}(x)$ defined in (1.9), then $V$ is a solution to (1.1) with the initial datum

$$
V(x, 0)=V_{0}(x) \equiv \begin{cases}v_{+}, & x<0 \\ v_{-}, & x>0\end{cases}
$$

where $v_{-}=-v_{+}$and $v_{+} \equiv|\bar{c}| \geq 0$. It is clear that $U$ satisfies $\mathrm{p} 2-\mathrm{p} 4$, whenever $V$ enjoys these properties. In the sequel, we thus assume without loss of generality that $u_{-}=-u_{+}$and $u_{+}>0$.

It has been shown in [2, Lemma 3.1] that if $u_{0} \in L^{\infty}(\mathbb{R})$ is non-increasing, odd and convex on $(0,+\infty)$, then the solution $u$ of (1.1)-(1.2) shares these properties w.r.t. $x$, for all $t>0$. The proof is based on a splitting method and on the fact that the "odd, concave/convex" property is conserved by both the hyperbolic equation $u_{t}+u u_{x}$ and the fractal equation $u_{t}+\Lambda^{1} u=0$. The same proof works with minor modifications to show that if $u_{0}$ is non-decreasing, odd and convex on $(-\infty, 0)$, then these properties are preserved by problem (1.1)-(1.2). Details are left to the reader since in that case, no shock can be created by the Burgers part and the proof is even easier. By the hypothesis $u_{-}=-u_{+}<0$ made above, the initial datum in (1.9) is non-decreasing, odd and convex on $(-\infty, 0)$. We conclude that so is the profile $U(1)$. The proof of properties p3-p4 is now complete.

What is left to prove is the limit in property p2. By Theorem 2.2 , we have $U(t) \rightarrow$ $U_{0}$ in $L_{l o c}^{1}(\mathbb{R})$ as $t \rightarrow 0$. In particular, the convergence holds true a.e. along a subsequence $t_{n} \rightarrow 0$ as $n \rightarrow+\infty$ and there exists $\pm x_{ \pm}>0$ such that $U\left(x_{ \pm}, t_{n}\right) \rightarrow$ $u_{ \pm}$. By the self-similarity of $U$, we get $U\left(\frac{x_{ \pm}}{t_{n}}, 1\right) \rightarrow u_{ \pm}$as $n \rightarrow+\infty$. Since $\frac{x_{ \pm}}{t_{n}} \rightarrow$ $\pm \infty$ and $U(1)$ is non-decreasing, we deduce property p2.

6.2. Some technical lemmata. The last property of Theorem 1.7 is the most difficult part to prove. In this preparatory subsection, we state and prove technical results that shall be needed in our reasoning.

Lemma 6.1. Let $v \in L^{\infty}(\mathbb{R})$ be non-negative, even and non-increasing on $(0,+\infty)$. Assume that there exists $\ell>0$ such that for all $x_{0}>1 / 2$,

$$
\lim _{n \rightarrow+\infty} n^{-1} \int_{n\left(x_{0}-1 / 2\right)}^{n\left(x_{0}+1 / 2\right)} y^{2} v(y) d y=\ell .
$$

Then, we have $y^{2} v(y) \rightarrow|y| \rightarrow+\infty \ell$.

Proof. For all $x_{0}>1 / 2$, we have

$$
n^{-1} \int_{n\left(x_{0}-1 / 2\right)}^{n\left(x_{0}+1 / 2\right)} y^{2} v(y) d y \geq n^{2}\left(x_{0}-1 / 2\right)^{2} v\left(n\left(x_{0}+1 / 2\right)\right),
$$

thanks to the fact that $v$ is non-increasing on $(0,+\infty)$. Hence, we have

$$
n^{2}\left(x_{0}+1 / 2\right)^{2} v\left(n\left(x_{0}+1 / 2\right)\right) \leq \frac{n^{2}\left(x_{0}+1 / 2\right)^{2}}{n^{2}\left(x_{0}-1 / 2\right)^{2}} n^{-1} \int_{n\left(x_{0}-1 / 2\right)}^{n\left(x_{0}+1 / 2\right)} y^{2} v(y) d y .
$$


Taking the upper semi-limit, we get for all $x_{0}>1 / 2$

$$
\limsup _{n \rightarrow+\infty} n^{2}\left(x_{0}+1 / 2\right)^{2} v\left(n\left(x_{0}+1 / 2\right)\right) \leq \ell\left(\frac{x_{0}+1 / 2}{x_{0}-1 / 2}\right)^{2},
$$

thanks to (6.3). In the same way, one can show that for all $x_{0}>1 / 2$,

$$
\ell\left(\frac{x_{0}-1 / 2}{x_{0}+1 / 2}\right)^{2} \leq \liminf _{n \rightarrow+\infty} n^{2}\left(x_{0}-1 / 2\right)^{2} v\left(n\left(x_{0}-1 / 2\right)\right) .
$$

Moreover, for fixed $x_{0}>1 / 2$ and all $y \geq x_{0}+1 / 2$, there exists an unique integer $n_{y}$ such that

$$
n_{y}\left(x_{0}+1 / 2\right) \leq y<\left(n_{y}+1\right)\left(x_{0}+1 / 2\right) .
$$

Using again that $v$ is non-increasing on $[0,+\infty)$, we infer that

$$
\begin{aligned}
y^{2} v(y) & \leq\left(n_{y}+1\right)^{2}\left(x_{0}+1 / 2\right)^{2} v\left(n_{y}\left(x_{0}+1 / 2\right)\right), \\
& =\frac{\left(n_{y}+1\right)^{2}\left(x_{0}+1 / 2\right)^{2}}{n_{y}^{2}\left(x_{0}+1 / 2\right)^{2}} n_{y}^{2}\left(x_{0}+1 / 2\right)^{2} v\left(n_{y}\left(x_{0}+1 / 2\right)\right) .
\end{aligned}
$$

Notice that $n_{y} \rightarrow+\infty$ as $y \rightarrow+\infty$. Therefore, passing to the upper semi-limit as $y \rightarrow+\infty$ in the inequality above, one can show that for all $x_{0}>1 / 2$

$$
\limsup _{y \rightarrow+\infty} y^{2} v(y) \leq \ell\left(\frac{x_{0}+1 / 2}{x_{0}-1 / 2}\right)^{2},
$$

thanks to (6.4). In the same way, we deduce from (6.5) that for all $x_{0}>1 / 2$

$$
\ell\left(\frac{x_{0}-1 / 2}{x_{0}+1 / 2}\right)^{2} \leq \liminf _{y \rightarrow+\infty} y^{2} v(y)
$$

Letting finally $x_{0} \rightarrow+\infty$ in both inequalities above implies that

$$
\ell \leq \liminf _{y \rightarrow+\infty} y^{2} v(y) \leq \limsup _{y \rightarrow+\infty} y^{2} v(y) \leq \ell .
$$

Since $v$ is even, we have completed the proof of the lemma.

For all $r>0$, the operator $\Lambda^{1}$ is the sum of $\Lambda_{r}^{(0)}$ and $\Lambda_{r}^{(1)}$. As far as $\Lambda_{r}^{(1)}$ is concerned, we have the following lemma.

Lemma 6.2. Let $u \in L^{\infty}(\mathbb{R})$ be non-decreasing, odd and convex on $(-\infty, 0)$. Then, for the operator defined in (2.2), we have $\Lambda_{r}^{(1)} u \in L_{\text {loc }}^{1}\left(\mathbb{R}_{*}\right)$ together with the inequality

$$
\int_{|x|>R}\left|\Lambda_{r}^{(1)} u(x)\right| d x \leq \frac{4 G_{1} r}{R-r}\|u\|_{\infty}
$$

for all $R>r>0$.

Proof. The proof is divided into a sequence of steps.

Step 1: estimates of $u_{x}$. The convex function $u$ on $(-\infty, 0)$ is locally Lipschitzcontinuous on $(-\infty, 0)$ and a fortiori a.e. differentiable. Since $u(0)=0$, we have for $x<0$

$$
\left|u_{x}(x)\right| \leq\|u\|_{\infty}|x|^{-1}
$$

Remark that this estimate holds true for $x \in \mathbb{R}$ since $u$ is odd.

Step 2: estimates of $u_{x x}$. By convexity of $u, u_{x x}$ is a non-negative Radon measure on $(-\infty, 0)$ in the distribution sense. Hence, $u_{x} \in B V_{l o c}((-\infty, 0))$ satisfies 
$\int_{(\widetilde{x}, x]} u_{y y}(d y)=u_{x}(x)-u_{x}(\widetilde{x})$, for a.e. $\widetilde{x}<x<0$. Using (6.7) and letting $\widetilde{x} \rightarrow-\infty$, we conclude that for a.e. $x<0$

$$
\int_{(-\infty, x]} u_{y y}(d y)=u_{x}(x)
$$

thanks to the sup-continuity of non-negative measures. Again by (6.7) and oddity of $u_{x x}$, this shows for a.e. $x \neq 0$

$$
\int_{|y| \geq|x|}\left|u_{y y}\right|(d y) \leq 2\|u\|_{\infty}|x|^{-1}
$$

notice that by the inf-continuity of non-negative measures, this inequality holds for all $x \neq 0$.

Step 3: estimate of $\Lambda_{r}^{(1)} u$. Let us prove that $\Lambda_{r}^{(1)} u$ is well-defined by formula (2.2) for a.e. $x \neq 0$. By the preceding steps, we know that $u \in L^{\infty}(\mathbb{R}) \cap W_{\text {loc }}^{1, \infty}\left(\mathbb{R}_{*}\right)$ and $u_{x} \in B V_{l o c}\left(\mathbb{R}_{*}\right)$. By Taylor's formula (see Lemma C.2 in Appendix [C), we infer that for all $R>r>0$

$$
\begin{aligned}
I & \equiv \int_{|x|>R} \int_{|z| \leq r} \frac{\left|u(x+z)-u(x)-u_{x}(x) z\right|}{|z|^{2}} d x d z \\
& \leq \int_{|x|>R} \int_{|z| \leq r}|z|^{-2}\left|\int_{I_{x, z}}\right| x+z-y\left|u_{y y}(d y)\right| d x d z,
\end{aligned}
$$

where $I_{x, z} \equiv(x, x+z)$ if $z>0$ and $I_{x, z} \equiv(x+z, x)$ in the opposite case. Therefore, we see that

$$
\begin{aligned}
I & \leq \int_{|x|>R} \int_{|z| \leq r}|z|^{-1} \int_{I_{x, z}}\left|u_{y y}\right|(d y) d x d z \\
& =\int_{\mathbb{R}_{*}} \int_{\mathbb{R}}|z|^{-1} \mathbf{1}_{\{|z| \leq r\}} \int_{|x|>R} \mathbf{1}_{I_{x, z}}(y) d x\left|u_{y y}\right|(d y) d z,
\end{aligned}
$$

by integrating first w.r.t $x$; notice that all the integrands are measurable by Fubini's theorem, since the Radon measure $\left|u_{y y}\right|(d y)$ is $\sigma$-finite on $\mathbb{R}_{*}$. For fixed $(y, z) \in$ $\mathbb{R}_{*} \times \mathbb{R}$, we have

$$
\mathbf{1}_{\{|z| \leq r\}} \int_{|x|>R} \mathbf{1}_{I_{x, z}}(y) d x \leq|z| \mathbf{1}_{\{|z| \leq r\}} \mathbf{1}_{\{|y| \geq R-r\}}
$$

because the measure of the set $\left\{x: y \in I_{x, z}\right\}$ can be estimated by $|z|$, and if $|z| \leq r$, then $\mathbf{1}_{I_{x, z}}(y)=0$ for all $|x|>R$ whenever $|y|<R-r$. It follows that

$$
I \leq \int_{\mathbb{R}_{*}} \int_{\mathbb{R}} \mathbf{1}_{\{|z| \leq r\}} \mathbf{1}_{\{|y| \geq R-r\}}\left|u_{y y}\right|(d y) d z=2 r \int_{|y| \geq R-r}\left|u_{y y}\right|(d y) .
$$

Recalling the definition of $I$ above and estimate (6.9), we have shown that

$$
\int_{|x|>R} \int_{|z| \leq r} \frac{\left|u(x+z)-u(x)-u_{x}(x) z\right|}{|z|^{2}} d x d z \leq 4 r\|u\|_{\infty}(R-r)^{-1} .
$$

Fubini's theorem then implies that $\Lambda_{r}^{(1)} u(x)$ is well-defined by (2.2) for a.e. $|x|>$ $R>r$ by satisfying the desired estimate (6.6).

Step 4: local integrability on $\mathbb{R}_{*}$. Estimate (6.6) implies that $\Lambda_{r}^{(1)} u \in L_{\text {loc }}^{1}(\mathbb{R} \backslash$ $[-r, r])$. In fact, $\Lambda_{r}^{(1)} u$ is locally integrable on all $\mathbb{R}_{*}$. Indeed, simple computations show that for all $r>\widetilde{r}>0$

$$
\Lambda_{r}^{(1)} u+\Lambda_{r}^{(0)} u=\Lambda_{\widetilde{r}}^{(1)} u+\Lambda_{\widetilde{r}}^{(0)} u,
$$


since their difference evaluated at some $x$ is equal to $\int_{\widetilde{r} \leq|z| \leq r} \frac{-u_{x}(x) z}{|z|^{2}}$, which is null by oddity of the function $z \rightarrow-u_{x}(x) z$. By Step 3, it follows that $\Lambda_{r}^{(1)} u=$ $\Lambda_{\widetilde{r}}^{(1)} u+\Lambda_{\widetilde{r}}^{(0)} u-\Lambda_{r}^{(0)} u \in L_{l o c}^{1}(\mathbb{R} \backslash[-\widetilde{r}, \widetilde{r}])$, which completes the proof.

It is clear that $\Lambda_{r}^{(0)}$ maps $L^{\infty}(\mathbb{R})$ into $L^{\infty}(\mathbb{R})$ and if $\left\{u_{n}\right\}_{n \in \mathbb{N}}$ is uniformly essentially bounded and $u_{n} \rightarrow u$ in $L_{l o c}^{1}(\mathbb{R})$, then $\Lambda_{r}^{(0)} u_{n} \rightarrow \Lambda_{r}^{(0)} u$ in $L_{l o c}^{1}(\mathbb{R})$ as $n \rightarrow+\infty$.

Remark 6.3. Lemma 6.2 implies that $\Lambda^{1} u \in L_{\text {loc }}^{1}\left(\mathbb{R}_{*}\right)$ whenever $u \in L^{\infty}(\mathbb{R})$ is non-decreasing, odd and convex on $(-\infty, 0)$. This sum does not depend on $r>0$ by (6.11). Moreover, one sees from (6.10), Fubini's theorem and (2.1), that for all $\varphi \in \mathcal{D}\left(\mathbb{R}_{*}\right), \int_{\mathbb{R}} \varphi \Lambda^{1} u d x=\int_{\mathbb{R}} u \Lambda^{1} \varphi d x$. This means that this sum corresponds to the distribution fractional Laplacian of $u$ on $\mathbb{R}_{*}$.

We deduce from the previous lemma the following one

Lemma 6.4. Let $u \in C_{b}(\mathbb{R})$ be non-decreasing, odd and convex on $(-\infty, 0)$. Then, the function $\Lambda^{1} u \in L_{\text {loc }}^{1}\left(\mathbb{R}_{*}\right)$ satisfies for all $x_{0}>1 / 2$,

$$
\lim _{n \rightarrow+\infty} n^{-1} \int_{n\left(x_{0}-1 / 2\right)}^{n\left(x_{0}+1 / 2\right)}\left|\Lambda^{1} u(y)\right| d y=0 .
$$

Proof. By Remark 6.3, one has $\Lambda^{1} u \in L_{\text {loc }}^{1}\left(\mathbb{R}_{*}\right)$. Let $r>0$ be fixed. One has

$$
\begin{aligned}
& I_{n} \equiv n^{-1} \int_{n\left(x_{0}-1 / 2\right)}^{n\left(x_{0}+1 / 2\right)}\left|\Lambda^{1} u(y)\right| d y \\
& \leq n^{-1} \int_{n\left(x_{0}-1 / 2\right)}^{n\left(x_{0}+1 / 2\right)}\left|\Lambda_{r}^{(1)} u(y)\right| d y+n^{-1} \int_{n\left(x_{0}-1 / 2\right)}^{n\left(x_{0}+1 / 2\right)}\left|\Lambda_{r}^{(0)} u(y)\right| d y \\
& \leq \frac{4 G_{1} r}{n^{2}\left(x_{0}-1 / 2\right)-n r}\|u\|_{\infty}+\sup \left\{\left|\Lambda_{r}^{(0)}(y)\right|: n\left(x_{0}-1 / 2\right)<y<n\left(x_{0}+1 / 2\right)\right\}
\end{aligned}
$$

thanks to (6.6). Moreover, $\Lambda_{r}^{(0)} u$ is continuous, hence the supremum above is achieved at some $y_{n} \geq n\left(x_{0}-1 / 2\right)$; hence, one has

$$
I_{n} \leq \frac{4 G_{1} r}{n^{2}\left(x_{0}-1 / 2\right)-n r}\|u\|_{\infty}+G_{1} \int_{|z|>r} \frac{\left|u\left(y_{n}+z\right)-u\left(y_{n}\right)\right|}{|z|^{2}} d z,
$$

where $\lim _{n \rightarrow+\infty} y_{n}=+\infty$. Since $u$ is non-decreasing and bounded, it has a limit at infinity; the dominated convergence theorem then implies that the integral term above tends to zero as $n \rightarrow+\infty$. It follows that $\lim _{n \rightarrow+\infty} I_{n}=0$.

6.3. Proof of property p5 from Theorem 1.7. We assume again without loss of generality that $u_{-}=-u_{+}<0$, thanks to the transformation (6.1); hence, $U_{0} \in$ $L^{\infty}(\mathbb{R})$ is non-decreasing, odd and convex on $(-\infty, 0)$ and so is $U(t)$ for all $t>0$ by properties $\mathrm{p} 2-\mathrm{p} 4$ of Theorem 1.7. We proceed again in several steps.

Step 1: study of $\Lambda^{1} U$. Before deriving the equation satisfied by $U(1)$, we study $\Lambda^{1} U$.

Lemma 6.5. Let $\alpha=1$ and $U$ be the self-similar solution from Theorem 1.5 with initial datum $U_{0}$ in (1.9) for some $u_{-}=-u_{+}<0$. Then, for all $t \geq 0$, one has $\Lambda^{1} U(t) \in L_{\text {loc }}^{1}\left(\mathbb{R}_{*}\right)$. Moreover, $\Lambda^{1} U(t)$ converges toward $\Lambda^{1} U_{0}$ in $L_{\text {loc }}^{1}\left(\mathbb{R}_{*}\right)$ as $t \rightarrow 0$, where for all $x \neq 0$

$$
\Lambda^{1} U_{0}(x)=\frac{u_{+}-u_{-}}{2 \pi^{2}} x^{-1}
$$


Proof. By properties p2-p4 of Theorem 1.7 $U(t) \in L^{\infty}(\mathbb{R})$ is non-decreasing, odd and convex on $(-\infty, 0)$ for all $t \geq 0$. By Remark 6.3, $\Lambda^{1} U(t)$ and $\Lambda^{1} U_{0}$ belong to $L_{l o c}^{1}\left(\mathbb{R}_{*}\right)$. By taking $0<r<|x|$, simple computations show that

$$
\Lambda_{r}^{(1)} U_{0}(x)=0 \quad \text { and } \quad \Lambda_{r}^{(0)} U_{0}(x)=\frac{u_{+}-u_{-}}{2 \pi^{2}} x^{-1}
$$

so that

$$
\Lambda^{1} U_{0}(x)=\frac{u_{+}-u_{-}}{2 \pi^{2}} x^{-1}
$$

here, we have used the equalities $\Gamma(1)=1$ and $\Gamma(1 / 2)=\sqrt{\pi}$ in order to get $G_{1}=\left(2 \pi^{2}\right)^{-1}$ in (2.2)-(2.3). Moreover, Theorem 2.2 implies that $U(t) \rightarrow U_{0}$ as $t \rightarrow 0$ in $L_{\text {loc }}^{1}(\mathbb{R})$ with $\|U(t)\|_{\infty} \leq\left\|U_{0}\right\|_{\infty}$. We remark that for fixed $r>$ $0, \Lambda_{r}^{(0)} U(t) \rightarrow \Lambda_{r}^{(0)} U_{0}$ in $L_{\text {loc }}^{1}(\mathbb{R})$ as $t \rightarrow 0$. It follows that for all $\widetilde{R}>R>r$,

$$
\begin{aligned}
& \limsup _{t \rightarrow 0} \int_{R<|x|<\widetilde{R}}\left|\Lambda^{1} U(t)-\Lambda^{1} U_{0}\right| d x \\
& \leq \limsup _{t \rightarrow 0} \int_{R<|x|<\widetilde{R}}\left|\Lambda_{r}^{(1)} U(t)-\Lambda_{r}^{(1)} U_{0}\right| d x, \\
& =\limsup _{t \rightarrow 0} \int_{R<|x|<\widetilde{R}}\left|\Lambda_{r}^{(1)} U(t)\right| d x \quad \text { by (6.12), } \\
& \leq \limsup _{t \rightarrow 0} 4 G_{1} r\|U(t)\|_{\infty}(R-r)^{-1} \text { by (6.6) in Lemma 6.2. } \\
& \leq 4 G_{1} r\left\|U_{0}\right\|_{\infty}(R-r)^{-1} .
\end{aligned}
$$

The proof is completed by letting $r \rightarrow 0$.

Step 2: equation satisfied by $U(1)$. By using $\eta(r)= \pm r$ in Definition 2.1, we obtain (in a classical way) that entropy solutions to (1.1) are distribution solutions, i.e.

$$
U_{t}+U U_{x}+\Lambda^{1} U=0 \quad \text { in } \quad \mathcal{D}^{\prime}(\mathbb{R} \times(0,+\infty)) .
$$

By property p1 of Theorem 1.7, one has $U(1) \in W^{1, \infty}(\mathbb{R})$. By the self-similarity $U(x, t)=U\left(\frac{x}{t}, 1\right)$, one has at least $U_{t}, U_{x} \in L_{\text {loc }}^{\infty}(\mathbb{R} \times(0,+\infty))$ together with the following equalities for a.e. $t>0$ and $x \in \mathbb{R}$

$$
U_{t}(x, t)=-x t^{-2} U_{x}\left(\frac{x}{t}, 1\right), \quad U_{x}(x, t)=t^{-1} U_{x}\left(\frac{x}{t}, 1\right) .
$$

By Lemma 6.5 we have also $\Lambda^{1} U(1) \in L_{l o c}^{1}\left(\mathbb{R}_{*}\right)$. Using again the self-similarity, it is easy to deduce from (2.1) that $\Lambda^{1} U \in L_{\text {loc }}^{1}\left(\mathbb{R}_{*} \times(0,+\infty)\right)$ with for a.e. $t>0$ and $x \in \mathbb{R}_{*}$,

$$
\Lambda^{1} U(x, t)=t^{-1} \Lambda^{1} U\left(\frac{x}{t}, 1\right)
$$

(in fact, $\Lambda^{1} U \in L_{\text {loc }}^{\infty}(\mathbb{R} \times(0,+\infty))$ by $(6.13)$ so that $\Lambda^{1} U(1) \in L_{\text {loc }}^{\infty}(\mathbb{R})$ ). Putting these formulas into (6.13), we get for a.e. $t>0$ and $x \in \mathbb{R}$,

$$
-x t^{-2} U_{x}\left(\frac{x}{t}, 1\right)+t^{-1} U\left(\frac{x}{t}, 1\right) U_{x}\left(\frac{x}{t}, 1\right)+t^{-1} \Lambda^{1} U\left(\frac{x}{t}, 1\right)=0 .
$$

Multiplying by $t$ and changing the variable by $y=t^{-1} x$, one infers that the profile $\mathcal{U}(y) \equiv U(y, 1)$ satisfies for a.e $y \in \mathbb{R}$

$$
(\mathcal{U}(y)-y) \mathcal{U}_{y}(y)+\Lambda^{1} \mathcal{U}(y)=0 .
$$

Step 3: reduction of the problem. By properties p1-p4, the function $\mathcal{U}_{y} \in L^{\infty}(\mathbb{R})$ is non-negative, even and non-decreasing on $(-\infty, 0)$. Then, Lemma 6.1 shows that 
the proof of p5 can be reduced to the proof of the following property:

$$
\forall x_{0}>1 / 2 \quad \lim _{n \rightarrow+\infty} n^{-1} \int_{n\left(x_{0}-1 / 2\right)}^{n\left(x_{0}+1 / 2\right)} y^{2} \mathcal{U}_{y}(y) d y=\frac{u_{+}-u_{-}}{2 \pi^{2}}
$$

Moreover, equality (6.14) implies that $\mathcal{U}_{y}(y)=\frac{\Lambda^{1} \mathcal{U}(y)}{y-\mathcal{U}(y)}$ (for a.e. $y>\|\mathcal{U}\|_{\infty}$ ) and Lemma 6.4 implies that

$$
\lim _{n \rightarrow+\infty} n^{-1} \int_{n\left(x_{0}-1 / 2\right)}^{n\left(x_{0}+1 / 2\right)}\left|\Lambda^{1} \mathcal{U}(y)\right| d y=0
$$

hence, since $\frac{y^{2}}{y-\mathcal{U}(y)}=y+\mathcal{O}(1)$ as $|y| \rightarrow+\infty$, one deduces that (6.15) is equivalent to the following property:

$$
\forall x_{0}>1 / 2 \quad \lim _{n \rightarrow+\infty} n^{-1} \int_{n\left(x_{0}-1 / 2\right)}^{n\left(x_{0}+1 / 2\right)} y \Lambda^{1} \mathcal{U}(y) d y=\frac{u_{+}-u_{-}}{2 \pi^{2}} .
$$

Conclusion: proof of (6.16). Let us change the variable by $y=n x$. Easy computations show that

$$
\begin{aligned}
n^{-1} \int_{n\left(x_{0}-1 / 2\right)}^{n\left(x_{0}+1 / 2\right)} y \Lambda^{1} \mathcal{U}(y) d y & =n^{-1} \int_{x_{0}-1 / 2}^{x_{0}+1 / 2} n x \Lambda^{1} U\left(\frac{x}{n^{-1}}, 1\right) n d x \\
& =\int_{x_{0}-1 / 2}^{x_{0}+1 / 2} x \Lambda^{1} U\left(x, n^{-1}\right) d x
\end{aligned}
$$

Since lemma 6.5 implies that $\left\{\Lambda^{1} U\left(x, n^{-1}\right)\right\}_{n \in \mathbb{N}}$ converges in $L^{1}\left(\left(x_{0}-1 / 2, x_{0}+1 / 2\right)\right)$ toward $\frac{u_{+}-u_{-}}{2 \pi^{2}}$ as $n \rightarrow+\infty$, the proofs of (6.16) and thus of property p5 are complete.

6.4. Duhamel's representation of the self-similar profile. It remains to prove Theorem 1.8, for which we need the following result.

Proposition 6.6. Let $\alpha=1$ and let $U$ be the self-similar solution of Theorem 1.5 with $u_{ \pm}= \pm 1 / 2$. Then, for all $x \in \mathbb{R}$, we have

$$
\begin{aligned}
& U(x, 1)=-1 / 2+H_{1}(x, 1) \\
& -\int_{0}^{1 / 2} \partial_{x} p_{1}(1-\tau) * \frac{U^{2}(\cdot / \tau, 1)}{2}(x) d \tau \\
& \quad-\int_{1 / 2}^{1} \tau^{-1} p_{1}(1-\tau) *\left(U(\cdot / \tau, 1) U_{x}(\cdot / \tau, 1)\right)(x) d \tau
\end{aligned}
$$

(where $\left.H_{1}(x, 1)=\int_{-\infty}^{x} p_{1}(y, 1) d y\right)$.

Proof. The proof proceeds in several steps.

Step 1: Duhamel's representation of the approximate solution. Notice that formula (6.17) makes sense. Indeed, by the homogeneity property (3.5), we have for all $t>0$

$$
\left\|\partial_{x} p_{1}(t)\right\|_{1}=C_{0} t^{-1}
$$

where $C_{0} \equiv\left\|\partial_{x} P_{1}(1)\right\|_{1}$ is finite by (3.6). Hence, the integral $\int_{0}^{1 / 2} \ldots d \tau$ in (6.17) is well-defined since the integration variable $\tau$ is far from the singularity at $\tau=1$. In the same way, since $U(1) \in W^{1, \infty}(\mathbb{R})$, the integral $\int_{1 / 2}^{1} \ldots d \tau$ is also well-defined.

Let now $u^{\varepsilon}$ be the solution to the regularized equation (3.1), with initial datum $U_{0}$ in (1.9). The goal is to pass to the limit in formula (3.3) at time $t=1$, 
namely

$$
\begin{aligned}
u^{\varepsilon}(x, 1) & =S_{1}^{\varepsilon}(1) U_{0}(x) \\
- & \int_{0}^{1 / 2} p_{2}(\varepsilon(1-\tau)) * \partial_{x} p_{1}(1-\tau) * \frac{\left(u^{\varepsilon}(\tau)\right)^{2}}{2}(x) d \tau \\
& \quad-\int_{1 / 2}^{1} p_{2}(\varepsilon(1-\tau)) * p_{1}(1-\tau) *\left(u^{\varepsilon}(\tau) u_{x}^{\varepsilon}(\tau)\right)(x) d \tau,
\end{aligned}
$$

for all $x \in \mathbb{R}$.

Step 2: pointwise limits and bounds of the integrands. We first remark that

$$
\lim _{x \rightarrow \pm \infty} u^{\varepsilon}(x, t)=u^{ \pm} .
$$

Indeed, we know that $u^{\varepsilon}$ is non-decreasing and it can be shown for instance that $u^{\varepsilon}-U_{0} \in L^{1}(\mathbb{R})$. This fact can be proved by splitting methods for instance.

Hence, thanks to Dini theorem for cumulative distribution functions, we know that for fixed $t>0, \lim _{\varepsilon \rightarrow 0} u^{\varepsilon}(t)$ converges toward $U(t)$ uniformly on $\mathbb{R}$.

Let us next recall that $\partial_{x} p_{1}(t) \in L^{1}(\mathbb{R})$, so that for fixed $\tau \in(0,1)$

$$
\lim _{\varepsilon \rightarrow 0} \partial_{x} p_{1}(1-\tau) * \frac{\left(u^{\varepsilon}(\tau)\right)^{2}}{2}=\partial_{x} p_{1}(1-\tau) * \frac{(U(\tau))^{2}}{2} \quad \text { uniformly on } \mathbb{R} .
$$

It follows from classical approximate unit properties of the heat kernel $p_{2}(x, t)$ that for all $\tau \in(0,1)$,

(6.20) $\lim _{\varepsilon \rightarrow 0} p_{2}(\varepsilon(1-\tau)) * \partial_{x} p_{1}(1-\tau) * \frac{\left(u^{\varepsilon}(\tau)\right)^{2}}{2}=\partial_{x} p_{1}(1-\tau) * \frac{(U(\tau))^{2}}{2}$

uniformly on $\mathbb{R}$. In particular, for all $\tau \in(0,1)$, we have also

$$
\lim _{\varepsilon \rightarrow 0} p_{2}(\varepsilon(1-\tau)) * p_{1}(1-\tau) *\left(u^{\varepsilon}(\tau) u_{x}^{\varepsilon}(\tau)\right)=p_{1}(1-\tau) *\left(U(\tau) U_{x}(\tau)\right)
$$

uniformly on $\mathbb{R}$, since

$$
\begin{aligned}
p_{2}(\varepsilon(1-\tau)) * \partial_{x} p_{1}(1-\tau) * \frac{\left(u^{\varepsilon}(\tau)\right)^{2}}{2} & \\
& =p_{2}(\varepsilon(1-\tau)) * p_{1}(1-\tau) *\left(u^{\varepsilon}(\tau) u_{x}^{\varepsilon}(\tau)\right)
\end{aligned}
$$

and $\partial_{x} p_{1}(1-\tau) * \frac{U^{2}(\tau)}{2}=p_{1}(1-\tau) *\left(U(\tau) U_{x}(\tau)\right)$.

Moreover, by (3.7), (3.8) with $p=+\infty$ and (6.18), one can see that the integrands of 6.19) are pointwise bounded by

$$
\left\|p_{2}(\varepsilon(1-\tau)) * \partial_{x} p_{1}(1-\tau) * \frac{\left(u^{\varepsilon}(\tau)\right)^{2}}{2}\right\|_{\infty} \leq C_{0}(1-\tau)^{-1} \frac{\left\|u_{0}\right\|_{\infty}^{2}}{2}
$$

and

$$
\left\|p_{2}(\varepsilon(1-\tau)) * p_{1}(1-\tau) *\left(u^{\varepsilon}(\tau) u_{x}^{\varepsilon}(\tau)\right)\right\|_{\infty} \leq \tau^{-1}\left\|u_{0}\right\|_{\infty} .
$$

Step 3: passage to the limit. Recall that

$$
\lim _{\varepsilon \rightarrow 0} S_{1}^{\varepsilon}(1) U_{0}=S_{1}(1) U_{0}=p_{1}(1) * U_{0}
$$

in $L^{p}(\mathbb{R})$ for all $p \in[1,+\infty]$. Let us recall that $U_{0}(x)= \pm 1 / 2$ for $\pm x \geq 0$ and $\int_{\mathbb{R}} p_{1}(y, 1) d y=1$, so that for all $x \in \mathbb{R}$

$$
1 / 2+p_{1}(1) * U_{0}(x)=p_{1}(1) *\left(U_{0}+1 / 2\right)(x)=\int_{-\infty}^{x} p_{1}(y, 1) d y=H_{1}(x, 1) .
$$

We have proved in particular that $\lim _{\varepsilon \rightarrow 0} S_{1}^{\varepsilon}(1) U_{0}=-1 / 2+H_{1}(1)$ pointwise on $\mathbb{R}$. 
In order to pass to the limit in the integral terms of (6.19), we use the Lebesgue dominated convergence theorem. We deduce from (6.20) and (6.22) that for all $x \in$ $\mathbb{R}$, the first integral term converges toward

$$
\int_{0}^{1 / 2} \partial_{x} p_{1}(1-\tau) * \frac{(U(\tau))^{2}}{2}(x) d \tau
$$

as $\varepsilon \rightarrow 0$. In the same way, we deduce from (6.21) and (6.23) that the last integral term converges toward

$$
\int_{1 / 2}^{1} p_{1}(1-\tau) *\left(U(\tau) U_{x}(\tau)\right)(x) d \tau .
$$

The limit as $\varepsilon \rightarrow 0$ in (6.19) then implies that for all $x \in \mathbb{R}$,

$$
\begin{aligned}
U(x, 1)=-1 / 2+H_{1}(x, 1)-\int_{0}^{1 / 2} \partial_{x} p_{1}(1 & -\tau) * \frac{U^{2}(\tau)}{2}(x) d \tau \\
& -\int_{1 / 2}^{1} p_{1}(1-\tau) *\left(U(\tau) U_{x}(\tau)\right)(x) d \tau .
\end{aligned}
$$

This completes the proof of (6.17), thanks to the self-similarity of $U$.

Proof of Theorem 1.8. We have to prove that for all $r>0$

$$
\mathbb{P}(|X-\bar{c}|<r)<\mathbb{P}(|Y-0|<r) .
$$

Let us verify that $\bar{c}$ and 0 are the medians of $X$ and $Y$, respectively. First, a simple computation allows to see that $p_{1}(x, 1)$, defined by Fourier transform by $\widehat{p_{1}}(\xi, 1)=$ $e^{-|\xi|}$, also satisfies formula (1.12). This density of probability is even and the median of $Y$ is null. Second, by property p3 of Theorem [1.7. $U_{x}(1)$ is symmetric w.r.t. to the axis $\{x=\bar{c}\}$ and the median of $X$ is $\bar{c}=\frac{u_{-}+u_{+}}{2}$.

In particular, the centered random variable $X-\bar{c}$ admits a density being the even function

$$
f_{X-\bar{c}}(x)=U_{x}(x+\bar{c}, 1) .
$$

It becomes clear that (6.24) is equivalent to the following property

$$
\forall x>0 \quad F_{X-\bar{c}}(x)<F_{Y}(x),
$$

where $F_{X-\bar{c}}$ and $F_{Y}$ are the cumulative distribution functions of $X-\bar{c}$ and $Y$, respectively.

Let us compute these functions. First, we have seen above that $f_{X-\bar{c}}(x)=$ $V_{x}(x, 1)$, where $V$ is defined by the transformation (6.1). Let us recall that $V$ is the self-similar solution to (1.1) with initial datum $V(x, 0)= \pm 1 / 2$ for $\pm x>0$. Hence, $F_{X-\bar{c}}$ is equal to $V(\cdot, 1)$ up to an additive constant, which has to be $1 / 2$ by property $\mathrm{p} 2$ of Theorem [1.7 that is to say, we have $F_{X-\bar{c}}(x)=1 / 2+V(x, 1)$ for all $x \in \mathbb{R}$. Second, we defined $H_{1}$ in Proposition 6.6 such that $F_{Y}(x)=H_{1}(x, 1)$. By this proposition, we have for all $x \in \mathbb{R}$,

$$
F_{X-\bar{c}}(x)=F_{Y}(x)-g(x),
$$

where $g(x)$ is defined by

$$
\begin{aligned}
g(x) \equiv \int_{0}^{1 / 2} \partial_{x} p_{1}(1-\tau) & * \frac{V^{2}(\cdot / \tau, 1)}{2}(x) d \tau \\
& +\int_{1 / 2}^{1} \tau^{-1} p_{1}(1-\tau) *\left(V(\cdot / \tau, 1) V_{x}(\cdot / \tau, 1)\right)(x) d \tau .
\end{aligned}
$$


One concludes that the proof of (6.25), and thus of (6.24), is equivalent to the proof of the positivity of $g(x)$ for positive $x$. But, by definition of $g$, it suffices to prove that for each $\tau \in(0,1)$ and $x>0$,

$$
p_{1}(1-\tau) *\left(V(\cdot / \tau, 1) V_{x}(\cdot / \tau, 1)\right)(x)>0 .
$$

Indeed, the second integral term in 6.26 would be positive, and the first integral term also, since for fixed $\tau$,

$$
\partial_{x} p_{1}(1-\tau) * \frac{V^{2}(\cdot / \tau, 1)}{2}(x)=\tau^{-1} p_{1}(1-\tau) *\left(V(\cdot / \tau, 1) V_{x}(\cdot / \tau, 1)\right)(x) .
$$

Let us end by proving inequality (6.27), thus concluding Theorem 1.8. It is clear that the function $V(\cdot / \tau, 1) V_{x}(\cdot / \tau, 1)$ is odd, since $V(1)$ is odd. Moreover, we already know that $V_{x}(1)$ is non-negative, even and non-increasing on $(0,+\infty)$, since $V(1)$ is non-decreasing, odd and concave on $[0,+\infty)$. By property p5, we conclude that $V_{x}(1)$ is positive a.e. on $(0,+\infty)$, and thus on $\mathbb{R}$ as even function. In particular, $V(1)$ is increasing and for all $x>0, V(x, 1)>V(0,1)=0$.

To summarize, $V(\cdot / \tau, 1) V_{x}(\cdot / \tau, 1)$ is odd and positive on $(0,+\infty)$. Moreover, it is clear that $p_{1}(1-\tau)$ is positive, even and decreasing on $(0,+\infty)$, see (1.12). A simple computation then implies that the convolution product in (6.27) is effectively positive for positive $x$. The proof of Theorem 1.8 is complete.

\section{Appendix A. A Key estimate}

Here is an estimate from the lines of [14, Proof of Lemma 3.1].

Lemma A.1 (inspired from [14]). Let $\alpha \in(0,2]$ and let us consider a function $v$ such that for all $a>0, v \in C_{b}^{\infty}(\mathbb{R} \times(a,+\infty)) \cap L^{\infty}\left(0,+\infty ; L^{1}(\mathbb{R})\right)$. Assume that for all $p \in[2,+\infty)$ and $t>0$,

$$
\int_{\mathbb{R}} v_{t}|v|^{p-2} v d x+\int_{\mathbb{R}}|v|^{p-2} v \Lambda^{\alpha} v d x \leq 0 .
$$

Then there is a constant $C=C(\alpha)>0$ such that for all $p \in[1,+\infty]$ and all $t>0$

$$
\|v(t)\|_{p} \leq C t^{-\frac{1}{\alpha}\left(1-\frac{1}{p}\right)}\|v\|_{L^{\infty}\left(0,+\infty ; L^{1}\right)} .
$$

The proof is based on the so-called Nash and Strook-Varopoulos inequalities.

Lemma A.2 (Nash inequality). Let $\alpha>0$. There exists a constant $C_{N}>0$ such that for all $w \in L^{1}(\mathbb{R})$ satisfying $\Lambda^{\alpha / 2} w \in L^{2}(\mathbb{R})$, one has

$$
\|w\|_{2}^{2(1+\alpha)} \leq C_{N}\left\|\Lambda^{\alpha / 2} w\right\|_{2}^{2}\|w\|_{1}^{2 \alpha} .
$$

Lemma A.3 (Strook-Varopoulos inequality). Let $\alpha \in(0,2]$. For all $p \in[2,+\infty)$ and $w \in L^{p-1}(\mathbb{R})$ satisfying $\Lambda^{\alpha} w \in L^{\infty}(\mathbb{R})$, one has

$$
\int_{\mathbb{R}}|w|^{p-2} w \Lambda^{\alpha} w d x \geq \frac{4(p-1)}{p^{2}} \int_{\mathbb{R}}\left(\Lambda^{\alpha / 2}|w|^{p / 2}\right)^{2} d x .
$$

Remark A.4. (1) In the case $\alpha=2$, simple computations show that one has an equality in place of an inequality.

(2) As suggested by the proof below, the second lemma is valid for all $p \in$ $[1,+\infty)$ with $w, \Lambda^{\alpha} w \in L^{p}(\mathbb{R})$, as well as in the muldimensional case and for more general operator $\Lambda^{\alpha}$ satisfies the postive maximum principle (see [10]).

Proofs and references for these results can be found in 14, 13. Let us give them for the sake of completeness. 
Proof of Lemma A.2. Let us first prove the result for $\varphi \in \mathcal{D}(\mathbb{R})$. By Plancherel equality, one has

$$
\|\varphi\|_{2}^{2}=\|\hat{\varphi}\|_{2}^{2} \leq \int_{|\xi|<r}|\hat{\varphi}(\xi)|^{d} \xi+r^{-\alpha} \int_{|\xi| \geq r}|\xi|^{\alpha}|\hat{\varphi}(\xi)|^{2} d \xi,
$$

for all $r>0$. Then, one gets

$$
\|\varphi\|_{2}^{2} \leq 2 r\|\hat{\varphi}\|_{\infty}^{2}+r^{-\alpha}\left\|\Lambda^{\alpha / 2} \varphi\right\|_{2}^{2} \leq 2 r\|\varphi\|_{1}^{2}+r^{-\alpha}\left\|\Lambda^{\alpha / 2} \varphi\right\|_{2}^{2} .
$$

Now an optimization w.r.t. $r>0$ gives the result for $\varphi$ smooth. The result for $w$ as in the lemma is deduced by approximation.

Proof of LemmaA.3. Let us proceed in several steps.

Step 1: a first inequality. Let us prove that for all $\beta, \gamma>0$ such that $\beta+\gamma=2$, one has for all non-negative reals $a, b$

$$
\left(a^{\beta}-b^{\beta}\right)\left(a^{\gamma}-b^{\gamma}\right) \geq \beta \gamma(a-b)^{2} .
$$

Let us assume without loss of generality that $a>b>0$ and $\beta \leq \gamma$. Developping each members of (A.3), one sees that this equation is equivalent to

$$
\begin{aligned}
(1-\beta \gamma) & \left(a^{2}+b^{2}\right) \\
& \stackrel{?}{\geq} a^{\beta} b^{\gamma}+a^{\gamma} b^{\beta}-2 \beta \gamma a b=(a b)^{\beta}\left(a^{2(1-\beta)}+b^{2(1-\beta)}-2 \beta \gamma(a b)^{1-\beta}\right) .
\end{aligned}
$$

Since one has $1-\beta \gamma=(1-\beta)^{2}$ and

$$
a^{2(1-\beta)}+b^{2(1-\beta)}-2 \beta \gamma(a b)^{1-\beta}=\left(a^{1-\beta}-b^{1-\beta}\right)^{2}+2(1-\beta \gamma)(a b)^{1-\beta},
$$

one deduces that (A.3) is equivalent to

$$
(1-\beta)^{2}\left(a^{2}+b^{2}-2 a b\right)=(1-\beta)^{2}(a-b)^{2} \stackrel{?}{\geq}(a b)^{\beta}\left(a^{1-\beta}-b^{1-\beta}\right)^{2} ;
$$

that is to say, one has to prove that for all $\beta \in(0,1]$ and $a>b>0$

$$
(1-\beta)(a-b) \stackrel{?}{\geq}(a b)^{\beta / 2}\left(a^{1-\beta}-b^{1-\beta}\right) .
$$

Dividing by $b>0$ and denoting $x$ the variable $\frac{a}{b}$, one has to prove that for all $\beta \in$ $(0,1]$ and $x>1$

$$
g(x) \equiv(1-\beta)(x-1)-x^{1-\beta / 2}+x^{\beta / 2} \stackrel{?}{\geq} 0 .
$$

Since $g$ is continuous w.r.t. $x \in[1,+\infty)$ with $g(1)=0$, it suffices to prove that $g^{\prime}(x) \geq$ 0 for all $x>1$. One has

$$
g^{\prime}(x)=1-\beta-(1-\beta / 2) x^{-\beta / 2}+\frac{\beta}{2} x^{-1+\beta / 2} .
$$

Again $g^{\prime}$ is continuous with $g(1)=0$, so that the proof of A.3 reduces finally to the proof of the non-negativity of $g^{\prime \prime}(x)$ for all $x>1$. One has

$$
g^{\prime \prime}(x)=\frac{\beta}{2}(1-\beta / 2) x^{-1-\beta / 2}+\frac{\beta}{2}(-1+\beta / 2) x^{-2+\beta / 2},
$$

so that $g^{\prime \prime}(x) \geq 0$ is equivalent to $x^{1-\beta} \stackrel{?}{\geq} 1$, which is true for $\beta \in(0,1]$ and $x>1$. The proof of (A.3) is complete. 
Conclusion. Take $\psi \in C_{c}(\mathbb{R})$ and assume $\psi \geq 0$. For all $r>0$ and $\beta, \gamma>0$, one has

$$
\begin{aligned}
& \int_{\mathbb{R}} \psi^{\gamma} \Lambda_{r}^{(0)} \psi^{\beta} d x \\
& =G_{\alpha} \iint_{|x-y|>r} \frac{\left(\psi^{\beta}(x)-\psi^{\beta}(y)\right) \psi^{\gamma}(x)}{|x-y|^{1+\alpha}} d x d y \\
& =G_{\alpha} \iint_{|x-y|>r} \frac{\left(\psi^{\beta}(y)-\psi^{\beta}(x)\right) \psi^{\gamma}(y)}{|x-y|^{1+\alpha}} d x d y
\end{aligned}
$$

by changing the variable $(x, y) \rightarrow(y, x)$ and using the fact that the measure $\frac{d x d y}{|x-y|}$ is symmetric. It follows that

$$
\int_{\mathbb{R}} \psi^{\gamma} \Lambda_{r}^{(0)} \psi^{\beta} d x=\frac{G_{\alpha}}{2} \iint_{|x-y|>r} \frac{\left(\psi^{\beta}(y)-\psi^{\beta}(x)\right)\left(\psi^{\gamma}(y)-\psi^{\gamma}(x)\right)}{|x-y|^{1+\alpha}} d x d y .
$$

On using Step 1, one deduces that for all $\psi \in C_{c}(\mathbb{R}), \psi \geq 0$, all $\beta, \gamma>0, \beta+\gamma=2$ and all $t>0$, one has

$$
\int_{\mathbb{R}} \psi^{\gamma} \Lambda_{r}^{(0)} \psi^{\beta} d x \geq \beta \gamma \int_{\mathbb{R}} \psi \Lambda_{r}^{(0)} \psi d x
$$

Take now $\varphi \in \mathcal{D}(\mathbb{R}), \varphi \geq 0$ and $p>1$. Let us choose $\psi=\varphi^{p / 2}, \beta=2 / p$ and $\gamma=2-\beta=2\left(1-\frac{1}{p}\right)$. Equation (A.4) gives:

$$
\int_{\mathbb{R}} \varphi^{p-1} \Lambda_{r}^{(0)} \varphi d x \geq \frac{4(p-1)}{p^{2}} \int_{\mathbb{R}} \varphi^{p / 2} \Lambda_{r}^{(0)} \varphi^{p / 2} d x .
$$

Hence, for $\varphi \in \mathcal{D}(\mathbb{R})$ not necessarily non-negative, Kato inequality (with $\eta(\cdot) \equiv$ $|\cdot|$ convex) implies

$$
\begin{aligned}
& \int_{\mathbb{R}}|\varphi|^{p-2} \varphi \Lambda_{r}^{(0)} \varphi d x \\
& \geq \int_{\mathbb{R}}|\varphi|^{p-1} \Lambda_{r}^{(0)}|\varphi| d x, \\
& \geq \frac{4(p-1)}{p^{2}} \int_{\mathbb{R}}|\varphi|^{p / 2} \Lambda_{r}^{(0)}|\varphi|^{p / 2} d x, \\
& =\frac{4(p-1)}{p^{2}} \frac{G_{\alpha}}{2} \iint_{|x-y|>r} \frac{\left(|\varphi|^{p / 2}(y)-|\varphi|^{p / 2}(x)\right)^{2}}{|x-y|^{1+\alpha}} d x d y .
\end{aligned}
$$

Passing to the limit as $r \rightarrow 0$, one concludes that

$$
\begin{aligned}
& \int_{\mathbb{R}}|\varphi|^{p-2} \varphi \Lambda^{\alpha} \varphi d x \\
& \geq \frac{G_{\alpha}}{2} \int_{\mathbb{R}} \int_{\mathbb{R}} \frac{\left(|\varphi|^{p / 2}(y)-|\varphi|^{p / 2}(x)\right)^{2}}{|x-y|^{1+\alpha}} d x d y, \\
& =\frac{4(p-1)}{p^{2}} \int_{\mathbb{R}}\left(\Lambda^{\alpha / 2}|\varphi|^{p / 2}\right)^{2} d x .
\end{aligned}
$$

This proves the result for $\varphi \in \mathcal{D}(\mathbb{R})$ non-negative. The proof for $w$ as in the lemma is complete by approximation.

Before proving Lemma A.1 one needs to establish a relationship between the differential inequality (A.1) and the $L^{p}$-norm in space of $v$ : 
Lemma A.5. Let $v$ such that $v \in C_{b}^{\infty}(\mathbb{R} \times(a,+\infty)) \cap L^{\infty}\left((0,+\infty) ; L^{1}(\mathbb{R})\right)$ for all $a>0$. Then for all $p \in[2,+\infty)$, the function $t>0 \rightarrow\|v(t)\|_{p}^{p}$ is locally Lipschitz-continuous with for a.e. $t>0$

$$
\frac{1}{p} \frac{d}{d t}\|v(t)\|_{p}^{p}=\int_{\mathbb{R}} v_{t}(x, t)|v(x, t)|^{p-2} v(x, t) d x .
$$

Proof. Let $\left\{\varphi_{n}\right\}_{n \in \mathbb{N}} \in \mathcal{D}(\mathbb{R} \times(0,+\infty))$ be a sequence such that

$$
\left\{\begin{array}{l}
\lim _{n} \varphi_{n}=v \text { in } C^{k}(K) \text { for all compact } K \subset \mathbb{R} \times(0,+\infty) \text { and } k \in \mathbb{N}, \\
\left\{\varphi_{n}\right\}_{n \in \mathbb{N}} \text { is bounded in } C^{k}(\mathbb{R} \times(a,+\infty)) \text { for all } a>0 \text { and } k \in \mathbb{N}, \\
\left|\varphi_{n}\right| \leq|v| \text { for all } n \in \mathbb{N} .
\end{array}\right.
$$

(such a sequence is easily constructed by taking $\varphi_{n} \equiv v \theta_{n}$, with $0 \leq \theta_{n} \leq 1, \theta_{n} \rightarrow 1$ in $C^{k}(K)$ and $\left\{\theta_{n}\right\}_{n \in \mathbb{N}}$ bounded in $\left.C^{k}(\mathbb{R} \times(a,+\infty))\right)$. One has for all $p \in[2,+\infty)$ and $t, s>0$,

$$
\frac{\left\|\varphi_{n}(t)\right\|_{p}^{p}-\left\|\varphi_{n}(s)\right\|_{p}^{p}}{p}=\int_{\mathbb{R}} \int_{s}^{t}\left|\varphi_{n}\right|^{p-2} \varphi_{n} \partial_{\tau} \varphi_{n} d x d \tau .
$$

By the dominated convergence theorem, one gets

$$
\begin{aligned}
& \lim _{n \rightarrow+\infty} \frac{\left\|\varphi_{n}(t)\right\|_{p}^{p}-\left\|\varphi_{n}(s)\right\|_{p}^{p}}{p} \\
= & \lim _{n \rightarrow+\infty} \int_{\mathbb{R}} \int_{s}^{t}\left|\varphi_{n}\right|^{p-2} \varphi_{n} \partial_{\tau} \varphi_{n} d x d \tau, \\
= & \int_{\mathbb{R}} \int_{s}^{t} v_{\tau}|v|^{p-2} v d x d \tau .
\end{aligned}
$$

But the dominated convergence theorem also allows to prove that

$$
\lim _{n \rightarrow+\infty} \frac{\left\|\varphi_{n}(t)\right\|_{p}^{p}-\left\|\varphi_{n}(s)\right\|_{p}^{p}}{p}=\frac{\|v(t)\|_{p}^{p}-\|v(s)\|_{p}^{p}}{p} .
$$

By uniqueness of the limit, one deduces that

$$
\frac{\|v(t)\|_{p}^{p}-\|v(s)\|_{p}^{p}}{p}=\int_{s}^{t}\left(\int_{\mathbb{R}} v_{\tau}|v|^{p-2} v d x\right) d \tau .
$$

Since $\tau \rightarrow \int_{\mathbb{R}} v_{\tau}(x, \tau)|v(x, \tau)|^{p-2} v(x, \tau) d x$ is bounded outside all neighborhood of $\tau=0$, the proof is complete.

Proof of Lemma A.1. The proof follows 14, Proof of Lemma 3.1]. One deduces from (A.1) and Lemmata A.3 and A.5 that for all $p \in[2,+\infty)$ and a.e. $t>0$,

$$
\frac{d}{d t}\|v(t)\|_{p}^{p}+4\left(1-\frac{1}{p}\right) \int_{\mathbb{R}}\left(\Lambda^{\alpha / 2}|v|^{p / 2}\right)^{2} d x \leq 0 .
$$

Let us now prove (A.2) for $p=2^{n}$ by induction on $n \geq 1$. In the sequel, $C_{0}$ denotes the constant $\|v\|_{L^{\infty}\left(0,+\infty, L^{1}\right)}$. For $p=2$, one uses A.6. and Lemma A.2 to get:

$$
\frac{d}{d t}\|v(t)\|_{2}^{2}+2 C_{N}^{-1} C_{0}^{-2 \alpha}\|v(t)\|_{2}^{2(1+\alpha)} \leq 0
$$

which leads to

$$
\|v(t)\|_{2} \leq C_{1} C_{0} t^{-\frac{1}{2 \alpha}} \quad \text { with } \quad C_{1} \equiv\left(\frac{C_{N}}{2 \alpha}\right)^{\frac{1}{2 \alpha}} .
$$

Suppose now that for $n \geq 2$ there is a constant $C_{n}$ such that for all $t>0$

$$
\|v(t)\|_{2^{n}} \leq C_{n} C_{0} t^{-\frac{1}{\alpha}\left(1-2^{-n}\right)} .
$$


Then, for $p=2^{n+1}, \underline{\mathrm{A} .6}$ and Lemma $\mathrm{A.2}$ applied to $w=v^{2^{n}}$ gives:

$$
\frac{d}{d t}\|v(t)\|_{2^{n+1}}^{2^{n+1}}+4\left(1-2^{-n-1}\right) C_{N}^{-1}\|v\|_{2^{n}}^{-2^{n+1} \alpha}\|v(t)\|_{2^{n+1}}^{2^{n+1}(1+\alpha)} \leq 0 .
$$

By the inductive hypothesis, one gets

$$
\frac{d}{d t}\|v(t)\|_{2^{n+1}}^{2^{n+1}}+4\left(1-2^{-n-1}\right) C_{N}^{-1}\left(C_{n} C_{0}\right)^{-2^{n+1} \alpha} t^{2^{n+1}-2}\left(\|v(t)\|_{2^{n+1}}^{2^{n+1}}\right)^{(1+\alpha)} \leq 0,
$$

which leads to

$$
\|v(t)\|_{2^{n+1}} \leq C_{n+1} C_{0} t^{-\frac{\left(1-2^{-n-1}\right)}{\alpha}} \quad \text { with } \quad C_{n+1}=C_{n}\left(\frac{C_{N}}{2 \alpha}\right)^{\frac{2^{-n-1}}{\alpha}}\left(2^{n 2^{-n-1}}\right)^{\frac{1}{\alpha}} .
$$

Now it rests to prove that $\lim _{\sup _{n \rightarrow+\infty}} C_{n}<+\infty$; indeed, the limit $n \rightarrow+\infty$ in the inequality above will gives (A.2) for $p=+\infty$ and the proof of the lemma will be complete by interpolation of the $L^{1}$ - and $L^{\infty}$-norms.

One has

$$
\ln C_{n+1}-\ln C_{n}=\ln \left(\frac{C_{n+1}}{C_{n}}\right)=\frac{2^{-n-1}}{\alpha} \ln \left(\frac{C_{N}}{2 \alpha}\right)+\frac{n 2^{-n-1}}{\alpha} \ln 2 \equiv u_{n},
$$

where the serie $\Sigma u_{n}$ is convergent. Summing up all these inequalities for $n=$ $1, \ldots, N$, one gets for all $N \geq 1, \ln C_{N+1}=\ln C_{1}+\Sigma_{n=1}^{N} u_{n}$. The limit $N \rightarrow+\infty$ then gives:

$$
\lim _{n \rightarrow+\infty} \ln C_{n}=\ln C_{1}+\Sigma_{k=1}^{+\infty} u_{k} \in \mathbb{R},
$$

so that $\lim _{n \rightarrow+\infty} C_{n}$ exits in $\mathbb{R}$

\section{Appendix B. Proof of Theorem 4.1}

Inequality from the following proposition is the starting point to prove Theorem 4.1

Proposition B.1. Let $u_{0}, \widetilde{u}_{0} \in L^{\infty}(\mathbb{R})$ and $\varepsilon>0$. Let $u^{\varepsilon}$ and $\widetilde{u^{\varepsilon}}$ be the solutions to (4.1) (4.2) with the initial data $u_{0}$ and $\tilde{u}_{0}$, resp. Then

$$
\int_{-R}^{R}\left|u^{\varepsilon}(x, t)-\widetilde{u^{\varepsilon}}(x, t)\right| d x \leq \int_{-R-L t}^{R+L t} S_{\alpha}^{\varepsilon}(t)\left|u_{0}-\widetilde{u}_{0}\right|(x) d x
$$

for all $t>0$ and $R>0$, where

$$
L=\max _{z \in[-M, M]}\left|f^{\prime}(z)\right| \text { and } \quad M=\max \left\{\left\|u_{0}\right\|_{\infty},\left\|\widetilde{u_{0}}\right\|_{\infty}\right\} .
$$

Even if this result does not appear in 1, its proof is based on ideas introduced in [1, Thm 3.2]. This is the reason why we only sketch the proof of Proposition B.1. the reader is referred to [1] for more details.

Sketch of proof of Proposition [B.1. The solution $u^{\varepsilon}$ of (4.1)-(4.2) satisfies

$$
\begin{aligned}
\int_{\mathbb{R}} \int_{a}^{+\infty} & \left(\eta\left(u^{\varepsilon}\right) \varphi_{t}+\phi\left(u^{\varepsilon}\right) \varphi_{x}\right) d x d t \\
& +\int_{\mathbb{R}} \int_{a}^{+\infty}\left(-\eta\left(u^{\varepsilon}\right) \Lambda_{r}^{(\alpha)} \varphi-\varphi \eta^{\prime}\left(u^{\varepsilon}\right) \Lambda_{r}^{(0)} u^{\varepsilon}\right) d x d t \\
& \quad-\varepsilon \int_{\mathbb{R}} \int_{a}^{+\infty}\left(\eta\left(u^{\varepsilon}\right)\right)_{x} \varphi_{x} d x d t+\int_{\mathbb{R}} \eta\left(u^{\varepsilon}(x, a)\right) \varphi(x, a) d x \geq 0
\end{aligned}
$$

for all $\varphi \in \mathcal{D}(\mathbb{R} \times[0,+\infty))$ non-negative, $\eta \in C^{2}(\mathbb{R})$ convex, $\phi^{\prime}=\eta^{\prime} f^{\prime}$ and $a, r>$ 0 . To show this inequality, it suffices to mutliply (4.1) by $\eta^{\prime}\left(u^{\varepsilon}\right) \varphi$, use the Kato inequalities (2.4) and integrate by parts over the domain $\mathbb{R} \times[a,+\infty)$. Now, let us 
introduce the so-called Kruzhkov entropy-flux pairs $\left(\eta_{k}, \phi_{k}\right)$ defined for fixed $k \in \mathbb{R}$ and all $u \in \mathbb{R}$ by

$$
\eta_{k}(u) \equiv|u-k| \quad \text { and } \quad \phi_{k}(u) \equiv \operatorname{sign}(u-k)(f(u)-f(k)),
$$

where "sign" denotes the sign function defined by

$$
\operatorname{sign}(u) \equiv \begin{cases}1, & u>0 \\ -1, & u<0 \\ 0, & u=0\end{cases}
$$

Consider a sequence $\left\{\eta_{k}^{n}\right\}_{n \in \mathbb{N}} \subset C^{2}(\mathbb{R})$ of convex functions converging toward $\eta_{k}$ locally uniformly on $\mathbb{R}$ and such that $\left(\eta_{k}^{n}\right)^{\prime} \rightarrow \operatorname{sign}(\cdot-k)$ pointwise on $\mathbb{R}$ by being bounded by 1 , as $n \rightarrow+\infty$. The associated fluxes $\phi_{k}^{n}(u) \equiv \int_{k}^{u} \eta_{k}^{\prime}(\tau) f^{\prime}(\tau) d \tau$ then converge toward $\phi_{k}$ pointwise on $\mathbb{R}$, as $n \rightarrow+\infty$, by being pointwise bounded by $\left|\phi_{k}^{n}(u)\right| \leq \operatorname{sign}(u-k) \int_{k}^{u}\left|f^{\prime}(\tau)\right| d \tau$. By the dominated convergence theorem, the passage to the limit in (B.3) with $(\eta, \phi)=\left(\eta_{k}^{n}, \phi_{k}^{n}\right)$ gives

$$
\begin{aligned}
& \int_{\mathbb{R}} \int_{a}^{+\infty}\left(\left|u^{\varepsilon}-k\right| \varphi_{t}+\operatorname{sign}\left(u^{\varepsilon}-k\right)\left(f\left(u^{\varepsilon}\right)-f(k)\right) \varphi_{x}\right) d x d t \\
& \quad+\int_{\mathbb{R}} \int_{a}^{+\infty}\left(-\left|u^{\varepsilon}-k\right| \Lambda_{r}^{(\alpha)} \varphi-\varphi \operatorname{sign}\left(u^{\varepsilon}-k\right) \Lambda_{r}^{(0)} u^{\varepsilon}\right) d x d t \\
& \quad-\varepsilon \int_{\mathbb{R}} \int_{a}^{+\infty} \operatorname{sign}\left(u^{\varepsilon}-k\right) u_{x}^{\varepsilon} \varphi_{x} d x d t+\int_{\mathbb{R}}\left|u^{\varepsilon}(x, a)-k\right| \varphi(x, a) d x \geq 0,
\end{aligned}
$$

for all $\varphi \in \mathcal{D}(\mathbb{R} \times[0,+\infty))$ non-negative, $a, r>0$ and $k \in \mathbb{R}$. In the same way, similar inequalities hold true for $\widetilde{u}^{\varepsilon}$.

On the basis of these inequalities, we claim that the well-known doubling variable technique of Kruzhkov allows us to compare $u_{\varepsilon}$ and $\widetilde{u}_{\varepsilon}$. To do so, we have to copy almost the same computations from [1, since the beginning of [1, Subsection 4.1] until [1, equation (4.11)] with $u=u^{\varepsilon}$ and $v=\widetilde{u^{\varepsilon}}$. The only difference comes from the term $-\varepsilon \int_{\mathbb{R}} \int_{a}^{+\infty} \operatorname{sign}\left(u^{\varepsilon}-k\right) u_{x}^{\varepsilon} \varphi_{x} d x d t$ in (B.4) and the term $-\varepsilon \int_{\mathbb{R}} \int_{a}^{+\infty} \operatorname{sign}\left(\widetilde{u^{\varepsilon}}-k\right) \widetilde{u_{x}^{\varepsilon}} \varphi_{x} d x d t$ in the entropy inequalities of $\widetilde{u^{\varepsilon}}$. But, these new terms do not present any particular difficulty, since $u_{\varepsilon}$ and $\widetilde{u}_{\varepsilon}$ are smooth. Arguing as in [1], one can show that for all $\phi \in \mathcal{D}(\mathbb{R} \times[0,+\infty))$ non-negative and $a>0$,

$$
\begin{aligned}
& \int_{\mathbb{R}} \int_{a}^{+\infty}\left|u^{\varepsilon}-\widetilde{u^{\varepsilon}}\right|\left(\phi_{t}+L\left|\phi_{x}\right|-\Lambda^{\alpha} \phi\right) d x d t \\
&-\varepsilon \int_{\mathbb{R}} \int_{a}^{+\infty} \operatorname{sign}\left(u^{\varepsilon}-\widetilde{u^{\varepsilon}}\right)\left(u^{\varepsilon}-\widetilde{u^{\varepsilon}}\right)_{x} \phi_{x} d x d t \\
& \quad+\int_{\mathbb{R}}\left|u^{\varepsilon}(x, a)-\widetilde{u^{\varepsilon}}(x, a)\right| \phi(x, a) d x \geq 0
\end{aligned}
$$

where $L$ is defined in (B.2). Since $\left|u^{\varepsilon}-\widetilde{u^{\varepsilon}}\right|$ is Lipschitz-continuous on $\mathbb{R} \times[a,+\infty)$, its a.e. derivative is equal to its distribution derivative with $\operatorname{sign}\left(u^{\varepsilon}-\widetilde{u^{\varepsilon}}\right)\left(u^{\varepsilon}-\widetilde{u^{\varepsilon}}\right)_{x}=$ $\left(\left|u^{\varepsilon}-\widetilde{u^{\varepsilon}}\right|\right)_{x}$. By integrating by parts, we deduce that

$$
\begin{aligned}
\int_{\mathbb{R}} \int_{a}^{+\infty}\left|u^{\varepsilon}-\widetilde{u^{\varepsilon}}\right|\left(\phi_{t}+L\left|\phi_{x}\right|-g[\phi]\right) d & \\
& +\int_{\mathbb{R}}\left|u^{\varepsilon}(x, a)-\widetilde{u^{\varepsilon}}(x, a)\right| \phi(x, a) d x \geq 0,
\end{aligned}
$$

where $g[\phi] \equiv\left(\Lambda^{\alpha}-\varepsilon \partial_{x}^{2}\right) \phi$. Passing to the limit as $a \rightarrow 0$, thanks to the continuity with values in $L_{l o c}^{1}(\mathbb{R})$ of $u^{\varepsilon}$ and $\widetilde{u^{\varepsilon}}$ in Theorem 3.1 , one can prove that for all 
non-negative $\phi \in \mathcal{D}(\mathbb{R} \times[0,+\infty))$

$$
\begin{aligned}
\int_{\mathbb{R}} \int_{0}^{+\infty}\left|u^{\varepsilon}-\widetilde{u^{\varepsilon}}\right|\left(\phi_{t}+L\left|\phi_{x}\right|-g[\phi]\right) & d x d t \\
& +\int_{\mathbb{R}}\left|u_{0}(x)-\widetilde{u}_{0}(x)\right| \phi(x, 0) d x \geq 0 .
\end{aligned}
$$

This is almost the same equation as that in [1, equation (4.11)] with the diffusive operator $g=\Lambda^{\alpha}-\varepsilon \partial_{x}^{2}$ instead of $g=\Lambda^{\alpha}$. Hence, we can argue exactly as in [1, Subsection 4.2] replacing the kernel of $\Lambda^{\alpha}$ by the kernel of the new operator $\Lambda^{\alpha}-$ $\varepsilon \partial_{x}^{2}$. This gives the desired inequality (B.1) in place of the inequality [1, equation $(3.1)]$

Proof of Theorem 4.1. Now, we are in a position to prove the convergence result in Theorem 4.1. The proof follows two steps: first we show the relative compactness of the family of functions $\mathcal{F} \equiv\left\{u^{\varepsilon}: \varepsilon \in(0,1]\right\}$ and, next, we pass to the limit in entropy inequalities.

Step 1: compactness. Let us prove that

$$
\mathcal{F} \text { is relatively compact in } F \equiv C\left([0, T] ; L^{1}([-R, R])\right)
$$

for all $T, R>0$. The space $F$ being a Banach space, the statement (B.6) is equivalent to the precompactness of $\mathcal{F}$ :

$$
\begin{gathered}
\forall \mu>0 \quad \exists \mathcal{F}_{\mu} \subseteq F \quad \text { relatively compact such that } \\
\lim _{\mu \rightarrow 0} \sup _{u^{\varepsilon} \in \mathcal{F}} \operatorname{dist}_{F}\left(u^{\varepsilon}, \mathcal{F}_{\mu}\right)=0 .
\end{gathered}
$$

To construct $\mathcal{F}_{\mu}$, we consider an approximation of the Dirac mass

$$
\rho_{\mu}(x) \equiv \mu^{-1} \rho\left(\mu^{-1} x\right)
$$

with a smooth, non-negative function $\rho=\rho(x)$, supported in $[-1,1]$ and such that $\int_{\mathbb{R}} \rho(x) d x=1$. Then we define

$$
\mathcal{F}_{\mu} \equiv\left\{u_{\mu}^{\varepsilon}: \varepsilon \in(0,1]\right\},
$$

where $u_{\mu}^{\varepsilon} \equiv u^{\varepsilon} *_{x} \rho_{\mu}$ and $*_{x}$ denotes the convolution product with respect to the space variable.

First, we have to prove that $\mathcal{F}_{\mu}$ is relatively compact in $F$. By estimate (3.7), it is clear that

$$
\left\|u_{\mu}^{\varepsilon}\right\|_{\infty} \leq\left\|u_{0}\right\|_{\infty} \quad \text { and } \quad\left\|\partial_{x} u_{\mu}^{\varepsilon}\right\|_{\infty} \leq\left\|u_{0}\right\|_{\infty}\left\|\partial_{x} \rho_{\mu}\right\|_{1} .
$$

Moreover, using equation (4.1) satisfied by $u^{\varepsilon}$ we obtain

$$
\partial_{t} u_{\mu}^{\varepsilon}=-\Lambda^{\alpha} u_{\mu}^{\varepsilon}+\varepsilon \partial_{x}^{2} u_{\mu}^{\varepsilon}-\left(f\left(u^{\varepsilon}\right)\right)_{x} *_{x} \rho_{\mu}=0 .
$$

Applying the equalities $\Lambda^{\alpha} u_{\mu}^{\varepsilon}=\Lambda^{\alpha}\left(u^{\varepsilon} *_{x} \rho_{\mu}\right)=u^{\varepsilon} *_{x}\left(\Lambda^{\alpha} \rho_{\mu}\right)$ we see that

$$
\left\|\Lambda^{\alpha} u_{\mu}^{\varepsilon}\right\|_{\infty} \leq\left\|u^{\varepsilon}\right\|_{\infty}\left\|\Lambda^{\alpha} \rho_{\mu}\right\|_{1} \leq\left\|u_{0}\right\|_{\infty}\left\|\Lambda^{\alpha} \rho_{\mu}\right\|_{1} .
$$

The same way, one can prove that

$$
\left\|\partial_{x} u_{\mu}^{\varepsilon}\right\|_{\infty} \leq\left\|u_{0}\right\|_{\infty}\left\|\partial_{x}^{2} \rho_{\mu}\right\|_{1} \text { and }\left\|\left(f\left(u^{\varepsilon}\right)\right)_{x} *_{x} \rho_{\mu}\right\|_{\infty} \leq C\left(\left\|u_{0}\right\|_{\infty}\right)\left\|\partial_{x} \rho_{\mu}\right\|_{1} .
$$

Consequently, it follows from equation (B.9) that for every fixed $\mu>0$, the time derivative of $u_{\mu}^{\varepsilon}$ is bounded independently of $\varepsilon \in(0,1]$. By (B.8) and the AscoliArzelà Theorem, we infer that $\mathcal{F}_{\mu}$ is relatively compact in $C_{b}([-R, R] \times[0, T])$ and, a fortiori, in $F$.

Next, we have to prove that $\lim _{\mu \rightarrow 0} \sup _{u^{\varepsilon} \in \mathcal{F}} \operatorname{dist}_{F}\left(u^{\varepsilon}, \mathcal{F}_{\mu}\right)=0$. Applying Theorem B.1 to the following simple inequality

$$
\left\|u^{\varepsilon}(t)-u_{\mu}^{\varepsilon}(t)\right\|_{L^{1}([-R, R])} \leq \int_{-R}^{R} \int_{-\mu}^{\mu}\left|u^{\varepsilon}(x, t)-u^{\varepsilon}(x-y, t)\right| \rho_{\mu}(y) d x d y
$$


we get

$$
\begin{aligned}
\left\|u^{\varepsilon}(t)-u_{\mu}^{\varepsilon}(t)\right\|_{L^{1}([-R, R])} & \leq \sup _{|y| \leq \mu} \int_{-R}^{R}\left|u^{\varepsilon}(x, t)-u^{\varepsilon}(x-y, t)\right| d x, \\
& \leq \sup _{|y| \leq \mu} \int_{-R-L t}^{R+L t} S_{\alpha}^{\varepsilon}(t) v_{0}^{y}(x) d x,
\end{aligned}
$$

where $v_{0}^{y}(x)=\left|u_{0}(x)-u_{0}(x-y)\right|$. Consequently, by Lemma C.1 in Appendix C, we see that there exists a modulus of continuity $\omega$ such that for all $r>0$ and $\varepsilon \in(0,1]$

$$
\left\|u^{\varepsilon}-u_{\mu}^{\varepsilon}\right\|_{F} \leq \sup _{|y| \leq \mu} \int_{-R-L T-r}^{R+L T+r} v_{0}^{y}(x) d x+\left\|v_{0}^{y}\right\|_{\infty} \omega(1 / r) .
$$

The continuity of the translation in $L^{1}$ implies that

$$
\lim _{\mu \rightarrow 0} \sup _{|y| \leq \mu} \int_{-R-L T-r}^{R+L T+r} v_{0}^{y}(x) d x=0 .
$$

Hence, it is clear that $\lim _{\mu \rightarrow 0} \sup _{\varepsilon \in(0,1]}\left\|u^{\varepsilon}-u_{\mu}^{\varepsilon}\right\|_{F}=0$, which proves (B.7) and thus (B.6).

Conclusion: passage to the limit. It follows from the first step that there exists $v \in C\left([0,+\infty) ; L_{l o c}^{1}(\mathbb{R})\right)$ such that $\lim _{\varepsilon \rightarrow 0} u^{\varepsilon}=v$ (up to a subsequence) in $C\left([0, T] ; L_{\text {loc }}^{1}(\mathbb{R})\right)$ for all $T>0$. Passing to another subsequence, if necessary, we can assume that $u^{\varepsilon} \rightarrow v$ a.e. From inequality (3.7), we deduce that $v \in L^{\infty}(\mathbb{R} \times(0,+\infty))$. What we have to prove is that $v=u$, however, by the uniqueness of entropy solutions ( $c f$. Theorem 2.2), it suffices to show that $v$ is an entropy solution to (2.7)-(2.8).

Let $\eta \in C^{2}(\mathbb{R})$ be convex, $\phi^{\prime}=\eta^{\prime} f^{\prime}$ and $r>0$. Integrating by parts the term $-\varepsilon \int_{\mathbb{R}} \int_{a}^{+\infty}\left(\eta\left(u^{\varepsilon}\right)\right)_{x} \varphi_{x} d x d t$ in (B.3) and passing to the limit $a \rightarrow 0$ in this inequality, we get

$$
\begin{gathered}
\int_{\mathbb{R}} \int_{0}^{+\infty}\left(\eta\left(u^{\varepsilon}\right) \varphi_{t}+\phi\left(u^{\varepsilon}\right) \varphi_{x}-\eta\left(u^{\varepsilon}\right) \Lambda_{r}^{(\alpha)} \varphi-\varphi \eta^{\prime}\left(u^{\varepsilon}\right) \Lambda_{r}^{(0)} u^{\varepsilon}\right) d x d t \\
+\int_{\mathbb{R}} \eta\left(u_{0}(x)\right) \varphi(x, 0) d x \geq-\varepsilon \int_{\mathbb{R}} \int_{0}^{+\infty} \eta\left(u^{\varepsilon}\right) \varphi_{x x} d x d t
\end{gathered}
$$

Finally, let us recall that $u^{\varepsilon} \rightarrow v$ a.e. as $\varepsilon \rightarrow 0$ and that $u^{\varepsilon}$ is bounded in $L^{\infty}$-norm by $\left\|u_{0}\right\|_{\infty}$. Hence, the Lebesgue dominated convergence theorem allows us to pass to the limit, as $\varepsilon \rightarrow 0$, in the inequality above and to deduce that

$$
\begin{gathered}
\int_{\mathbb{R}} \int_{0}^{+\infty}\left(\eta(v) \varphi_{t}+\phi(v) \varphi_{x}-\right. \\
\left.\eta(v) \Lambda_{r}^{(\alpha)} \varphi-\varphi \eta^{\prime}(v) \Lambda_{r}^{(0)} v\right) d x d t \\
+\int_{\mathbb{R}} \eta\left(u_{0}(x)\right) \varphi(x, 0) d x \geq 0 .
\end{gathered}
$$

Hence, according to Definition 2.1 and Theorem 2.2, the function $v$ is the unique entropy solution to (2.7)-(2.8). The proof of Theorem 4.1 is complete.

\section{Appendix C. Additional technical lemmata}

Lemma C.1. There exists a modulus of continuity $\omega$ such that for all $v_{0} \in L^{\infty}(\mathbb{R})$, all $T, R, r>0$, and all $\varepsilon \in(0,1]$, we have

$$
\sup _{t \in[0, T]} \int_{-R-L t}^{R+L t} S_{\alpha}^{\varepsilon}(t)\left|v_{0}\right|(x) d x \leq \int_{-R-L T-r}^{R+L T+r}\left|v_{0}(x)\right| d x+\left\|v_{0}\right\|_{\infty} \omega(1 / r) .
$$


Proof. First, we write

$$
\begin{aligned}
\sup _{t \in[0, T]} \int_{-R-L t}^{R+L t} S_{\alpha}^{\varepsilon}(t) \mid & v_{0} \mid(x) d x \\
& =\sup _{t \in[0, T]} \int_{-R-L t}^{R+L t} p_{\alpha}(t) * p_{2}(\varepsilon t) *\left|v_{0}\right|(x) d x \\
& \leq \sup _{s \in[0, T]} \sup _{t \in[0, T]} \int_{-R-L t}^{R+L t} p_{\alpha}(t) * p_{2}(\varepsilon s) *\left|v_{0}\right|(x) d x
\end{aligned}
$$

Now, for every $s \in[0, T]$, we estimate from above the following function

$$
M(s) \equiv \sup _{t \in[0, T]} \int_{-R-L t}^{R+L t} p_{\alpha}(t) * w_{0}(x) d x,
$$

where $w_{0} \equiv p_{2}(\varepsilon s) *\left|v_{0}\right|$. Using properties of the kernel $p_{\alpha}$ and its self-similarity (see (3.5) ) we obtain

$$
\begin{aligned}
\int_{-R-L t}^{R+L t} p_{\alpha}(t) * w_{0}(x) d x= & \int_{|x| \leq R+L t} \int_{|y| \leq r / 2} p_{\alpha}(y, t) w_{0}(x-y) d x d y \\
& +\int_{|x| \leq R+L t} \int_{|y| \geq r / 2} p_{\alpha}(y, t) w_{0}(x-y) d x d y \\
\leq & \left\|p_{\alpha}(t)\right\|_{1} \int_{-R-L t-r / 2}^{R+L t+r / 2}\left|w_{0}(x)\right| d x \\
& +\left\|w_{0}\right\|_{\infty} 2(R+L t) \int_{|y| \geq r / 2} p_{\alpha}(y, t) d y \\
= & \int_{-R-L t-r / 2}^{R+L t+r / 2}\left|w_{0}(x)\right| d x \\
& +\left\|w_{0}\right\|_{\infty} 2(R+L t) \int_{|x| \geq t^{-\frac{1}{\alpha}} r / 2} p_{\alpha}(x, 1) d x .
\end{aligned}
$$

Computing the supremum with respect to $t \in[0, T]$ we infer that

$$
M(s) \leq \int_{-R-L T-r / 2}^{R+L T+r / 2}\left|w_{0}(x)\right| d x+\left\|w_{0}\right\|_{\infty} \omega_{\alpha}(1 / r),
$$

where $\omega_{\alpha}:[0,+\infty) \rightarrow(0,+\infty)$ is defined by

$$
\omega_{\alpha}(1 / r) \equiv(2 R+2 L T) \int_{|x| \geq T^{-\frac{1}{\alpha}} r / 2} p_{\alpha}(x, 1) d x .
$$

It is clear that the modulus of continuity $\omega_{\alpha}$ is non-decreasing and satisfies

$$
\lim _{r \rightarrow+\infty} \omega_{\alpha}(1 / r)=0
$$

Finally, since $\left\|w_{0}\right\|_{\infty}=\left\|p_{2}(\varepsilon s) *\left|v_{0}\right|\right\|_{\infty} \leq\left\|v_{0}\right\|_{\infty}$, we obtain

$$
M(s) \leq \int_{-R-L T-r / 2}^{R+L T+r / 2}\left|w_{0}(x)\right| d x+\left\|v_{0}\right\|_{\infty} \omega_{\alpha}(1 / r) .
$$


Analogous computations show now that

$$
\begin{aligned}
\int_{-R-L T-r / 2}^{R+L T+r / 2}\left|w_{0}(x)\right| d x & =\int_{-R-L T-r / 2}^{R+L T+r / 2} p_{2}(\varepsilon s) *\left|v_{0}\right|(x) d x \\
& \leq \int_{-R-L T-r}^{R+L T+r}\left|v_{0}(x)\right| d x+\left\|v_{0}\right\|_{\infty} \omega_{2}(\sqrt{\varepsilon} / r) \\
& \leq \int_{-R-L T-r}^{R+L T+r}\left|v_{0}(x)\right| d x+\left\|v_{0}\right\|_{\infty} \omega_{2}(1 / r)
\end{aligned}
$$

because $\varepsilon \leq 1$.

Finally, with the new modulus of continuity $\omega(1 / r) \equiv \omega_{\alpha}(1 / r)+\omega_{2}(1 / r)$, we have

$$
M(s) \leq \int_{-R-L T-r}^{R+L T+r}\left|v_{0}(x)\right| d x+\left\|v_{0}\right\|_{\infty} \omega(1 / r) .
$$

Coming back to inequality (C.1), we complete the proof of Lemma C.1.

Lemma C.2. Let $I$ be an open interval of $\mathbb{R}$ and $u \in W^{1, \infty}(I)$ be such that $u_{x} \in$ $B V(I)$. Then, for a.e. $x \in I$ and all $z \in I-x$, we have

$$
u(x+z)=u(x)+u_{x}(x) z+\int_{I_{x, z}}|x+z-y| u_{y y}(d y),
$$

where $I_{x, z} \equiv(x, x+z)$ if $z>0$ and $I_{x, z} \equiv(x+z, x)$ if not.

Proof. We can reduce to the case $I=(a, b)$ with $a, b \in \mathbb{R}$. Let us assume without loss of generality that $z>0$. Since $u_{x} \in B V(I)$, the function $\widetilde{u}_{x}(x) \equiv$ $c+\int_{(a, x]} u_{y y}(d y)$ is an a.e. representative of $u_{x}$, where $c$ is the trace of $u_{x}$ on the left boundary of $I$. The trace of $u_{x} \in B V\left(I_{x, z}\right)$ onto $\{x\}$ is equal to $\widetilde{u}_{x}(x)$, because $\{x\}$ is the left boundary of $I_{x+z}$. Simple integration by parts formulas now give

$$
\begin{aligned}
u(x+z) & =u(x)+\int_{I_{x, z}} u_{y}(y) d y \\
& =u(x)-\int_{I_{x, z}}(y-x-z) u_{y y}(d y)+\widetilde{u}_{x}(x) z .
\end{aligned}
$$

The proof is complete.

\section{REFERENCES}

[1] N. Alibaud, Entropy formulation for fractal conservation laws, J. Evol. Equ. 7 (2007), 145175.

[2] N. Alibaud, J. Droniou and J. Vovelle, Occurrence and non-appearance of shock in fractal Burgers equations, J. Hyperbolic Differ. Equ 4 (2007), 479-499.

[3] P. Biler, G. Karch and W. Woyczyński, Asymptotics for multifractal conservation laws, Studia Math. 135 (1999), 231-252.

[4] P. Biler, G. Karch and W. A. Woyczyński, Asymptotics for conservation laws involving Lévy diffusion generators, Studia Math. 148 (2001), 171-192.

[5] P. Biler, G. Karch and W. A. Woyczyński, Critical nonliearity exponent and self-similar asymptotics for Lévy conservation laws, Ann. Inst. Henri Poincaré, Analyse non-linéaire, 18 (2001), 613-637.

[6] L. Brandolese and G. Karch,, Far field asymptotics of solutions of convection equations with anomalous diffusion, J. Evol. Equ., 8 (2008), 307-326.

[7] C. H. Chan and M. Czubak, Regularity of solutions for the critical $N$-dimensional Burgers equation, (2008), 1-31. arXiv:0810.3055 3 [math.AP].

[8] J. Droniou, T. Gallouët and J. Vovelle, Global solution and smoothing effect for a non-local regularization of a hyperbolic equation, J. Evol. Equ. 3 (2002), $499-521$.

[9] J. Droniou and C. Imbert, Fractal first order partial differential equations, Arch. Rat. Mech. Anal. 182 (2006), 299-331. 
[10] W. Hoh, Pseudo differential operators generating Markov processes, Habilitationsschrift, Bielefeld 1998, 154 pages.

[11] B. Jourdain, S. Méléard and W. Woyczyński, A probabilistic approach for nonlinear equations involving the fractional Laplacian and singular operator, Potential Analysis 23 (2005), 55-81.

[12] B.Jourdain, S.Méléard and W.Woyczyński, Probabilistic approximation and inviscid limits for one-dimensional fractional conservation laws, Bernoulli 11 (2005), 689-714.

[13] G. Karch, Non-linear evolution equations withoutanomalous diffusion to appear in Lecture notes of the Neas Center for Mathematical Modeling MATFYZPRESS Publishing House of the Faculty of Mathematics and Physics Charles University Prague.

[14] G. Karch, C. Miao and X. Xu, On convergence of solutions of fractal Burgers equation toward rarefaction waves, SIAM J. Math. Anal. 39 (2008), 1536-1549.

[15] K. Karlsen and S. Ulusoy, Stability of entropy solutions for Lévy mixed hyperbolic/parabolic equations, Preprint (2009).

[16] A. Kiselev, F. Nazarov and R. Shterenberg, Blow up and regularity for fractal Burgers equation, Dyn. Partial Differ. Equ. 5 (2008), 211-240.

[17] C. Miao, B. Yuan and B. Zhang, Well-posedness of the Cauchy problem for fractional power dissipative equations, Nonlinear Anal. 68 (2008), 461-484.

[18] C. Miao and G. Wu, Global well-posedness of the critical Burgers equation in critical Besov spaces, J. Differential Equations 247 (2009) 1673-1693.

N. Alibaud: Laboratoire de Mathématiques de Besançon, umR CNRS 6623, UniverSité de Franche-Comté, UFR Sciences et techniques, 16 route de Gray, 25030 BesanÇon CEDEX, FRANCE

E-mail address: Nathael.Alibaud@ens2m.fr

URL: http://www-math.univ-f comte.fr/pp_Annu/NALIBAUD

C. Imbert: CEREMAde, UMR CNRS 7534, Université Paris-Dauphine, Place de LatTRE DE TASsigny, 75775 PARIS CEDEX 16, France

E-mail address: imbert@ceremade.dauphine.fr

$U R L:$ http://www. ceremade.dauphine.fr/ imbert

G. Karch: Instytut Matematyczny, Uniwersytet WrocŁawski, Pl. Grunwaldzki 2/4, 50-384 WrocŁaW, Poland

E-mail address: Grzegorz.Karch@math.uni.wroc.pl

URL: http://www.math.uni.wroc.pl/ ${ }^{\sim} \mathrm{karch}$ 